\title{
Directed motion of membrane proteins under an entropy-driven potential field generated by anchored proteins
}

\author{
Yusheng Shen, ${ }^{1}$ Chengjie Luo, ${ }^{1}$ Yan Wen, ${ }^{1}$ Wei He $\odot,{ }^{1}$ Pingbo Huang, ${ }^{2}$ Hsuan-Yi Chen, ${ }^{3}$ Pik-Yin Lai, ${ }^{3}$ and Penger Tong ${ }^{1}$ \\ ${ }^{1}$ Department of Physics, Hong Kong University of Science and Technology, Clear Water Bay, Kowloon, Hong Kong, China \\ ${ }^{2}$ Division of Life Science, Hong Kong University of Science and Technology, Clear Water Bay, Kowloon, Hong Kong, China \\ ${ }^{3}$ Department of Physics and Center for Complex Systems, National Central University, Taoyuan City 320, Taiwan, Republic of China
}

(Received 19 April 2021; accepted 22 November 2021; published 20 December 2021)

\begin{abstract}
Directed transport of proteins and other molecules in a crowded living cell is often carried out by diffusion at short distances and by motor-driven cargo transport over long distances. Here we demonstrate, by both experiments and theory, that anchored proteins inside the cell can generate a spatially varying and temporally stable potential (free-energy) landscape for intracellular or membrane transport in the mesoscale. By using a micropatterned substrate, we introduce a periodic array of anchored integrins on the basal membrane of cultured Xenopus muscle cells. This patterned array of anchored integrins imposes a periodic potential $U(x)$ to the lateral motion of nicotinic acetylcholine receptors (AChRs) on the cell membrane. From a thorough analysis of a large volume of AChR trajectories obtained over a wide range of sampling conditions and long durations from 385 cells, we find the trapping potential $U(x)$ and its effects on the drift velocity $V_{x}(x)$ and diffusion coefficient $D_{x}(x)$ of AChRs. Our findings suggest that anchored proteins may play an essential role in generating an effective potential landscape to guide molecular motion in the mesoscale ranging from protein trapping and directed motion to enhanced protein-protein interactions over a long range.
\end{abstract}

DOI: 10.1103/PhysRevResearch.3.043195

\section{INTRODUCTION}

A living cell is a densely packed compartment filled with individual proteins, lipids, and sugars in the cell cytoplasm, as well as the filamentous networks permeating the cell [1]. How a protein molecule wades through and functions in such a crowded environment is an outstanding issue in cell biology and biological physics, which has been under ongoing debates [2-6]. From a wealth of new data obtained in recent years, our general view of intracellular dynamics and transport has evolved into a new paradigm, in which two intracellular constituents play very different roles. One is an adenosine-triphosphate-(ATP)-dependent mobile component, such as molecular motors and ATP-driven polymerization forces, which provide active mechanisms for molecular transport, signaling, and control [6-9]. The other is an immobile or transiently confined component [10-13], such as various anchored proteins. Cellular ATP is also required to keep these proteins in place against random diffusion [14,15].

Compared to the rapid growth of knowledge on active transport and dynamics, our current understanding of anchored proteins is still primitive. For example, in the study of membrane diffusion of eukaryotic cells, one found that about half of the transmembrane proteins, corresponding to $10-20 \%$ of total membrane area, are bound to the underlying

Published by the American Physical Society under the terms of the Creative Commons Attribution 4.0 International license. Further distribution of this work must maintain attribution to the author(s) and the published article's title, journal citation, and DOI. cortical actin network and, therefore, are effectively immobile on timescales of minutes to hours [16]. These anchored proteins were assumed to be random obstacles in the membrane and hinder the motion of other mobile proteins [17-23]. The protein diffusion on a live cell membrane was found to be significantly reduced (up to 25 times) compared to that in artificially reconstituted membranes $[15,24]$. Up to now, we did not know why a living cell spends energy (ATP) to lock certain proteins in place, which in turn put a "brake" [2] on all other mobile proteins inside the cell or on the cell membrane. More recent studies [11,25-27] revealed that the anchored proteins are not evenly distributed on the membrane, as is commonly believed, but in an organized manner.

In this paper we demonstrate, by both experiments and theory, that a nonuniform concentration field $n(\mathbf{r})$ of anchored proteins can generate a spatially varying and temporally stable potential (free-energy) landscape $U(\mathbf{r})$ to other (nonmotor) mobile proteins in the region. This entropy-driven potential field offers a novel way for directed molecular transport in the mesoscale $(20 \mathrm{~nm}-1 \mu \mathrm{m})$, which complements the conventional methods of directed molecular transport by diffusion at short distances and motor-driven cargo transport over long distances. This finding suggests an important and biologically tunable mechanism for intracellular transport, protein trapping, and protein-protein interactions initiated not by specific chemical signaling, as is usually assumed, but by nonspecific physical forces that yield remarkably simple universal laws.

\section{EXPERIMENTAL RESULTS}

For the sake of simplicity, we first consider the anchored proteins having a one-dimensional (1D) concentration 
distribution $n(x)$ (area fraction). Because of the excluded volume effect of the anchored proteins, the Gibbs free energy (or chemical potential) of a mobile protein is increased by $-k_{B} T \ln [1-n(x)]\left[\simeq n(x) k_{B} T\right.$ for small $\left.n\right]$ due to the reduced available space. This gives rise to an entropy-driven potential field $U(x) \simeq n(x) k_{B} T$ for mobile proteins, pushing them to move from a high concentration region of anchored proteins to a low concentration region. Here $k_{B} T$ is the thermal energy of a mobile protein. This potential field can be readily generalized for a 2D distribution $n(x, y)$, and thus can generate a trapping potential (funnel) in the mesoscale with an energy barrier $E_{b} \sim k_{B} T$, as expected. Higher energy barriers can be obtained if the effects of the size and concentration of the anchored proteins and their interactions with mobile proteins are included (see more discussions in Sec. III below).

The anchored protein chosen for this study is integrin containing $\beta_{1}$ subunit (integrin for short), which facilitates cell-extracellular matrix (ECM) adhesion and is abundantly expressed on the membrane of muscle cells [25,28]. To alter the spatial distribution of anchored integrins, we use the technique of micropatterning [29] to construct a patterned substrate for cell culture. The patterned substrate consists of identical parallel microgrooves of height $=1 \mu \mathrm{m}$ and separation $\lambda=2 \mu \mathrm{m}$, which are made on a thin layer of poly(dimethylsiloxane) (PDMS) coated on a glass coverslip (see Secs. IV A and IV B below for more experimental details). To quantitatively study the effect of the patterned array of anchored integrins on other mobile transmembrane proteins, we follow the motion of nicotinic acetylcholine receptors (AChRs) on live muscle cell membrane cultured from embryos of Xenopus laevis (see Sec. IV C 1 below for more experimental details). AChR is a well-characterized neurotransmitter receptor for the study of neuromuscular junctions (NMJs) [13,30,31]. The lateral mobility of AChRs plays an essential role in determining the response of the postsynaptic membrane to neurotransmitter stimuli. In this study, we focus on AChRs because their lateral motion on a live muscle cell membrane cultured on a flat substrate has been carefully studied in recent experiments $[13,15]$. It was found that the motion of AChRs for cells cultured on a flat coverslip is isotropic without any preferred direction, and they have a high mobile ratio $(\simeq 65 \%$ ), which is defined as the number ratio of the mobile trajectories to the total number of AChR trajectories recorded.

As illustrated in Fig. 1(a), the microgroove pattern allows the integrins to predominantly anchor on the ridge, so that the resulting distribution of $\beta_{1}$-integrins shows a parallel-stripe pattern, as shown in Fig. 1(b). By comparing the fluorescent image of $\beta_{1}$-integrins with the bright-field image of microgrooves (see Sec. IV C 2 below for more experimental details), we find the parallel green stripes lie on top of the underlying microgroove ridges with the same spacing $\lambda$. Because of the anisotropy of the microgroove pattern, the cultured muscle cells are elongated along the microgrooves. Furthermore, the cortical F-actin network is also aligned along the trough of the microgrooves. Figures 1(b) and 1(c) reveal that the spatial distribution of the integrins and cortical Factin both exhibit a stripe pattern, but the stripe position is staggered. For muscle cells cultured on a flat glass coverslip, integrins are found to be randomly distributed across the basal (a)

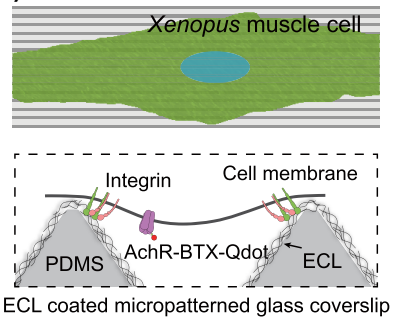

(b)

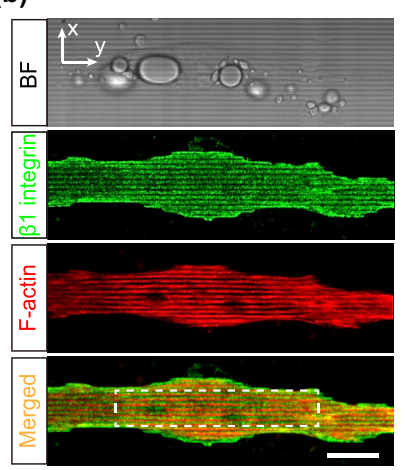

(c)

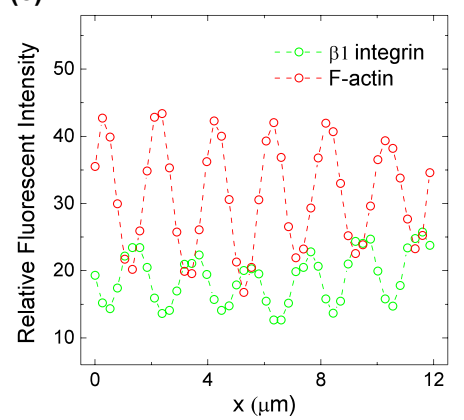

(d)

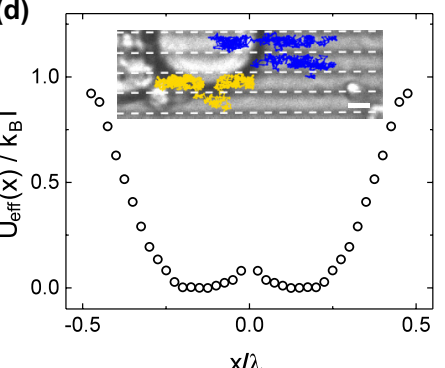

FIG. 1. Microgroove-patterned substrate induces a periodic array of $1 \mathrm{D}$ potential traps for AChRs. (a) A schematic top view of a Xenopus muscle cell (green) cultured on a 2- $\mu \mathrm{m}$-microgroovepatterned substrate (upper panel) and a magnified side view of the membrane-substrate interface showing the anchored integrins on the ridge and mobile AChRs diffusing over a single microgroove (lower panel). (b) Comparison of bright-field (BF) and costained fluorescent images of muscle cells cultured on a $2-\mu \mathrm{m}$-microgroove-patterned substrate. The fluorescent images, which are obtained by averaging the vertical $z$-sections of the basal membrane of $\sim 1 \mu \mathrm{m}$ thickness, show the spatial distribution of $\beta_{1}$-integrins (green), cortical F-actin (red), and their superposition (merged) on the basal membrane. The scale bar is $20 \mu \mathrm{m}$. (c) Relative fluorescent intensity of $\beta_{1}$-integrins (green) and cortical F-actin (red) (in arbitrary units) across the parallel microgrooves ( $x$-axis). The intensity profiles are averaged along the microgrooves ( $y$-axis) in the region as marked by the white dashed box in (b). (d) Measured effective trapping potential $U_{\text {eff }}(x)$ for mobile AChRs as a function of the normalized position $x / \lambda$ across a single microgroove with $\lambda=2 \mu \mathrm{m}$. Here $x / \lambda=0$ is set at the bottom of the microgroove. The inset shows two representative AChR trajectories (blue and yellow) with 1500 time steps (300 s) over the BF microgroove pattern. The white dashed lines indicate the ridge of the microgrooves. The scale bar is $2 \mu \mathrm{m}$.

membrane without any ordered pattern [see Fig. 6(a) below]. Figure 1 thus demonstrates our ability to use micropatterning to modulate/control the spatial distribution of the anchored integrins in a cell membrane.

In the experiment, the individual AChRs are labeled by bright and photostable fluorescent quantum dots (QDs) (see Sec. IV C 3 below for more experimental details). We obtain the AChR trajectories from consecutive images of the QDs, and we find their position $\mathbf{r}(t)$ (and hence the position of AChRs) at time $t$ using a homemade single-particle tracking program with a spatial resolution of $\sim 20 \mathrm{~nm}$ (see Sec. IV D below for more experimental details). Because the viscosity of the plasma membrane is approximately 500 times higher than that of the extracellular medium, the motion of the QD-labeled 

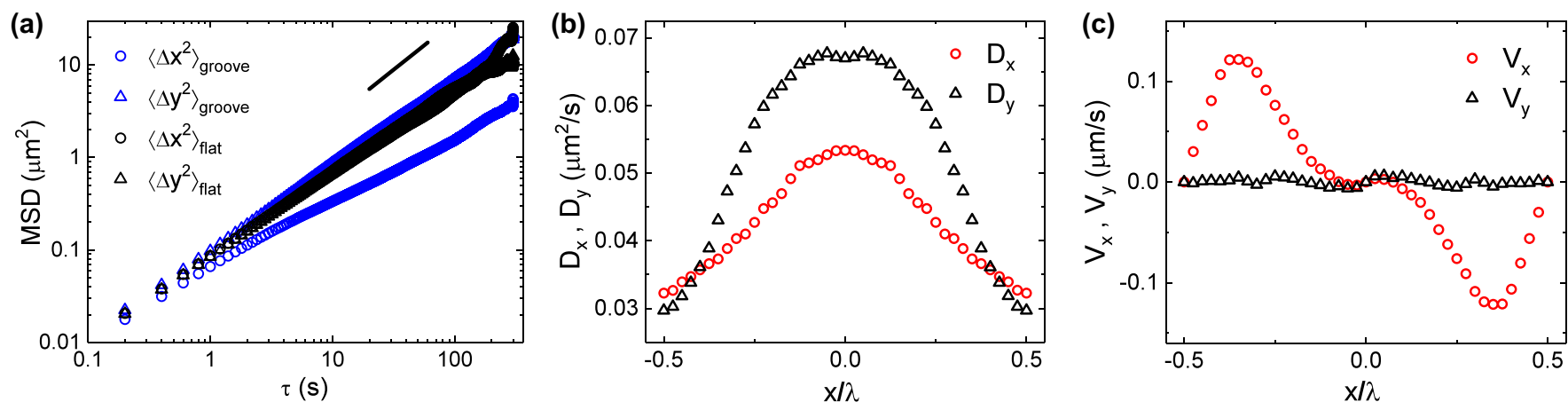

FIG. 2. Statistical properties of the displacements $\Delta x(\tau)$ and $\Delta y(\tau)$ for AChRs. (a) Measured MSDs $\left\langle\Delta x^{2}(\tau)\right\rangle$ (circles) and $\left\langle\Delta y^{2}(\tau)\right\rangle$ (triangles) as a function of delay time $\tau$ for muscle cells cultured on a 2- $\mu \mathrm{m}$-microgroove-patterned substrate (blue symbols) and on a flat substrate (black symbols). The solid line indicates the relationship $\left\langle\Delta x^{2}(\tau)\right\rangle \sim \tau$ with a slope of unity in the log-log plot. (b) Measured short-time diffusion coefficients $D_{x}$ (red circles) and $D_{y}$ (black triangles) as a function of $x / \lambda$ for muscle cells cultured on a 2- $\mu$ m-microgroovepatterned substrate. (c) Measured drift velocities $V_{x}$ (red circles) and $V_{y}$ (black triangles) as a function of $x / \lambda$ for muscle cells cultured on a $2-\mu \mathrm{m}$-microgroove-patterned substrate.

AChRs is determined primarily by their transmembrane domains [15,32]. With the help of the advanced single-molecule tracking algorithm, we are able to obtain a large volume of individual AChR trajectories from more than 385 live cells over a wide range of sampling rates (up to $80 \mathrm{~Hz}$ ) and long durations (up to $300 \mathrm{~s}$ ) (see Sec. IV D below for more experimental details). Moving in the patterned array of anchored integrins, the AChRs do not enjoy unrestricted lateral diffusion as was observed for cells cultured on a flat substrate $[13,15]$. Instead, the AChR trajectories reveal a confined diffusion within a single groove and occasional hopping to a neighboring groove, as shown in the inset of Fig. 1(d).

From the AChR trajectories, we obtain the probability density function (PDF) $H(x, y)$ of finding an AChR at location $(x, y)$, where the $x$-axis is normal to the microgroove and the $y$-axis is in parallel with the microgroove [see the coordinates shown in Fig. 1(b)]. By averaging the AChR trajectories along the microgroove ( $y$-axis) and over different grooves, we obtain the PDF $H(x)=\langle H(x, y)\rangle_{y}$, from which we define the effective potential field $U_{\text {eff }}(x)$ as $U_{\text {eff }}(x) / k_{B} T=-\ln H(x)$, where $H(x)$ is normalized in such a way that $U_{\text {eff }}(x)=0$ at the bottom of the potential well. As shown in Fig. 1(d), the obtained trapping potential $U_{\text {eff }}(x)$ across a single microgroove has a $\mathrm{U}$-shape with an energy barrier height $E_{b} \simeq 1.0 k_{B} T$. A unique advantage of using the parallel microgrooves is that it allows us to pick up targeted signals of AChRs with a specific spatial period $\lambda$ along the normal direction of the microgrooves and average out uncorrelated noise resulting from other unknown anchored proteins or interactions with the surroundings. With this lock-in technique in space, the spatial period $\lambda$ used is much smaller than the cell size [see Fig. 1(a)] so that one can average many AChR trajectories obtained from the bulk region of the cell membrane without any edge effect. Meanwhile, the value of $\lambda$ is large enough to avoid seeing peculiar effects of molecular complexes associated with anchored integrins, such as focal adhesion complexes [33].

From the obtained AChR trajectories, we also compute the statistics of the displacements, $\Delta x(\tau)=x(t+\tau)-x(t)$ across the microgroove and $\Delta y(\tau)=y(t+\tau)-y(t)$ along the microgroove. The statistical averages are taken both along the microgroove and over different grooves (see Sec. IV D below for more experimental details). As shown in Fig. 2(a), the measured mean-squared displacements (MSDs) $\left\langle\Delta x^{2}(\tau)\right\rangle$ and $\left\langle\Delta y^{2}(\tau)\right\rangle$ for cells cultured on a flat substrate overlap with each other and are a linear function of delay time $\tau$. This suggests that the motion of AChRs on a flat substrate is isotropic and can be well described by diffusion with a long-time ( $\tau>$ $1 \mathrm{~s})$ diffusion coefficient, $\left(D_{L}\right)_{y} \simeq 0.025 \mu \mathrm{m}^{2} / \mathrm{s}[13,15]$. The measured MSDs for cells cultured on a microgroove patterned substrate, on the other hand, exhibit an anisotropic diffusion behavior with the measured $\left\langle\Delta y^{2}(\tau)\right\rangle$ being nearly the same as those for a flat substrate. The measured $\left\langle\Delta x^{2}(\tau)\right\rangle$ in the $\log -\log$ plot is not a linear function and exhibits a crossover behavior from short-time diffusion $\left(\tau<\tau_{L} \simeq 1 \mathrm{~s}\right)$ to longtime diffusion $\left(\tau>\tau_{H} \simeq 50 \mathrm{~s}\right.$ ), with the long-time diffusion coefficient, $\left(D_{L}\right)_{x} \simeq 0.014 \mu \mathrm{m}^{2} / \mathrm{s}$, being much smaller than $\left(D_{L}\right)_{y}$. In the crossover region $\tau_{L} \lesssim \tau \lesssim \tau_{H}$, the motion of AChRs becomes heterogeneous and undergoes subdiffusion, $\left\langle\Delta x^{2}(\tau)\right\rangle \sim \tau^{\alpha}$, with $0.6 \lesssim \alpha \lesssim 0.8$. Such a crossover behavior of MSD has been observed previously for colloidal diffusion over potential barriers [13,35-37].

The local diffusion of AChRs reveals a strong $x$ dependence inside the potential trap. Here the local (or short-time) diffusion coefficients are defined as $D_{x}(x)=$ $\left\langle\Delta x^{2}\right\rangle /(2 \tau)$ and $D_{y}(x)=\left\langle\Delta y^{2}\right\rangle /(2 \tau)$, where the delay time $\tau$ is taken at the smallest value $\tau=0.2 \mathrm{~s}$. As shown in Fig. 2(b), the measured $D_{x}(x)$ reaches its maximal value at the bottom of the microgroove where the concentration of anchored integrins is minimal, as observed from the fluorescent intensity profile of the integrins [see Figs. 1(c) and 3(c) below]. The value of $D_{x}(x)$ is reduced by more than $40 \%$ at the ridge of the microgroove where the integrins are densely populated. The obtained $D_{y}(x)$ exhibits a similar profile. The fact that the measured maximal value of $D_{x}$ is significantly smaller than that of $D_{y}$ suggests that the alignment of the cortical F-actin network at the bottom of the microgroove also plays a role in hindering the motion of AChRs.

Similarly, we determine the drift velocities, $V_{x}(x)=$ $\langle\Delta x\rangle / \tau$ and $V_{y}(x)=\langle\Delta y\rangle / \tau$, with $\tau=0.2$ s. An intriguing effect of the potential trap is to produce a nonzero drift 


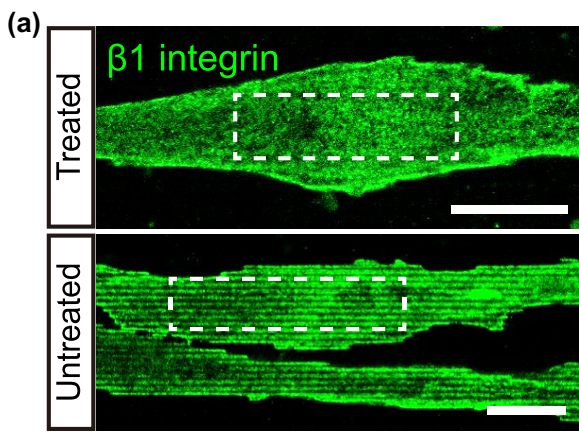

(b)

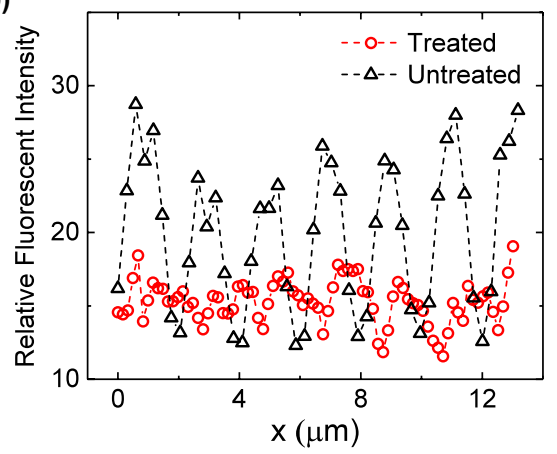

(d)

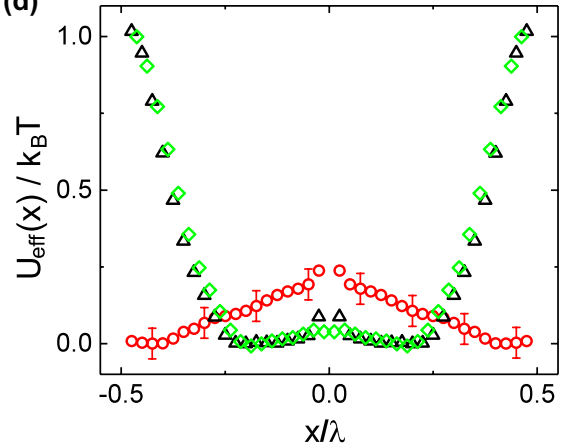

(e)

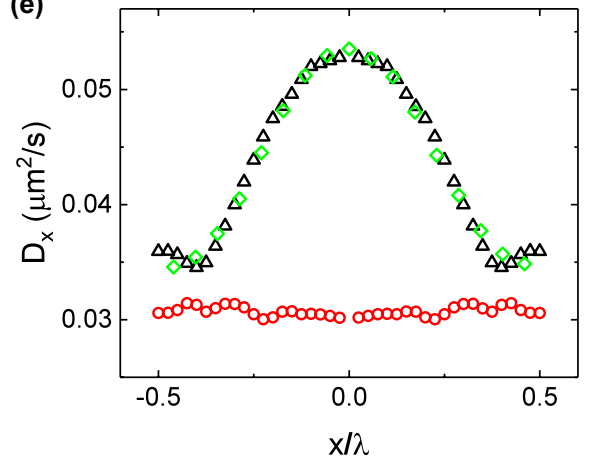

(c)

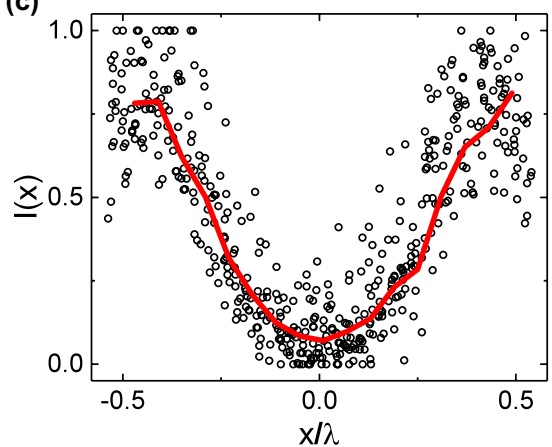

(f)

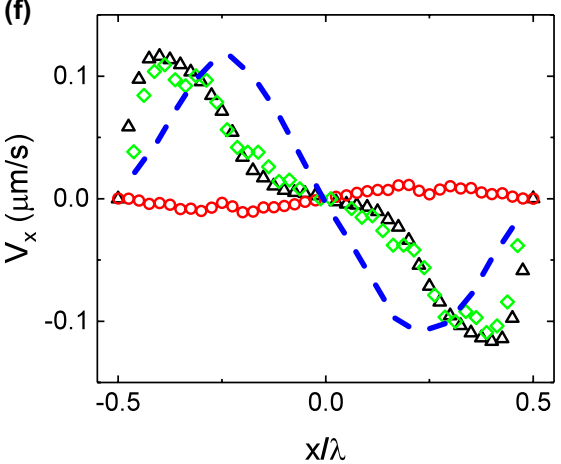

FIG. 3. Effects of the Matrigel treatment. (a) Comparison of fluorescent images of $\beta_{1}$-integrins (green) on the basal membrane for muscle cells with the Matrigel treatment (upper panel) and without the Matrigel treatment (lower panel). The scale bars are $20 \mu \mathrm{m}$. (b) Relative fluorescent intensity of $\beta_{1}$-integrins (in arbitrary units) across the parallel microgrooves ( $x$-axis) with (red circles) and without (black triangles) the Matrigel treatment. The intensity profiles are averaged along the microgrooves ( $y$-axis) in the regions as marked by the white dashed boxes in (a). (c) Normalized fluorescent intensity profile $I(x)$ of $\beta_{1}$-integrins as a function of $x / \lambda$ across a single microgroove. The values of $I(x)$ are normalized between 1 and 0 (black circles) and are obtained from different cells without the Matrigel treatment. The red solid line shows the mean profile $\langle I(x)\rangle$. (d)-(f) Measured (d) effective potential $U_{\text {eff }}(x)$, (e) short-time diffusion coefficient $D_{x}$, and (f) drift velocity $V_{x}$ as a function of $x / \lambda$. The comparison between the Matrigel-treated cells (red circles) and untreated cells (black triangles) is made using the same batch of cells cultured on a 2- $\mu \mathrm{m}$-microgroove-patterned substrate under the same conditions, except for the use of different culture media, one with Matrigel added $(0.45 \mathrm{mg} / \mathrm{mL})$ and the other without Matrigel. The green diamonds in (d) show the calculated $U_{\text {eff }}(x)$ using Eq. (6). The green diamonds in (e) show the calculated $D_{x}(x)$ using Eq. (7). The best-fit result is obtained with $D_{0}=0.03 \pm 0.005 \mu \mathrm{m}^{2} / \mathrm{s}, \alpha=0.55 \pm 0.05$, $\beta=0.95 \pm 0.05$, and $D_{\min }=0.028 \pm 0.005 \mu \mathrm{m}^{2} / \mathrm{s}$. The green diamonds in (f) show the calculated $V_{x}(x)$ using the first equality in Eq. (5). The blue dashed line in (f) shows the calculated $V_{x}(x)$ [or $V_{l}(x)$ ] using the second equality in Eq. (5) with $D_{0}=0.03 \mu \mathrm{m}^{2} / \mathrm{s}, \alpha=0.55$, and $\beta=0.95$.

velocity $V_{x}$ across the microgroove. As shown in Fig. 2(c), the measured $V_{x}$ reaches its maximal value $\left(V_{x}\right)_{m} \simeq 0.13 \mu \mathrm{m} / \mathrm{s}$ in the ridge region where the concentration of anchored integrins has a large gradient. The net movement of AChRs is always directed toward the bottom of the microgroove, where the concentration gradient of the integrins is close to zero and the resulting $V_{x}$ also reduces to a value close to zero. With this value of $\left(V_{x}\right)_{m}$, it will take approximately $8 \mathrm{~s}$ for the AChRs to move $1 \mu \mathrm{m}$, which is about 10 times faster than diffusion. Because the motion of AChRs along the microgroove is random, the drift velocity $V_{y}(x)$ along the $y$-axis is zero at any $x$-position. Figure 2(c) thus demonstrates that AChRs have a reduced diffusion coefficient in the crowded region of anchored integrins and tend to move to a less crowded region following the direction of the concentration gradient of the integrins. This gives rise to an effective way of using a potential gradient to produce directed motion of mobile proteins toward a low potential region.

Although the above potential traps in the plasma membrane are induced by a patterned substrate, they nevertheless are very much relevant to cell biology. First, the substrate-induced (nonuniform) distribution of anchored integrins can be altered by adding soluble extracellular matrix (ECM) proteins (Matrigel, $0.45 \mathrm{mg} / \mathrm{mL}$ ) in the culture medium (see Sec. IV C 1 below for more experimental details). These proteins adsorb on the outer side of the suspended cell membrane, allowing integrins to anchor in the suspended membrane [38-40]. As a result, the microgroove-induced stripe pattern of anchored integrins is erased and the spatial distribution of the $\beta_{1}$-integrins becomes uniform across the basal membrane without a visible pattern, as shown in Figs. 3(a) and 3(b) (and supplementary Fig. S1 [41]). The Matrigel treatment, therefore, gives us an effective key to turn off the effective trapping potential $U_{\text {eff }}(x)$ induced by anchored integrins without changing the shape of the cell membrane.

The membrane shape modulation on the microgroovepatterned substrate can be checked by measuring the fluorescent intensity variation of the QDs that are conjugated to the AChRs moving on the cell membrane. Because the focal plane of the microscope used in the experiment is always set at 
(a)

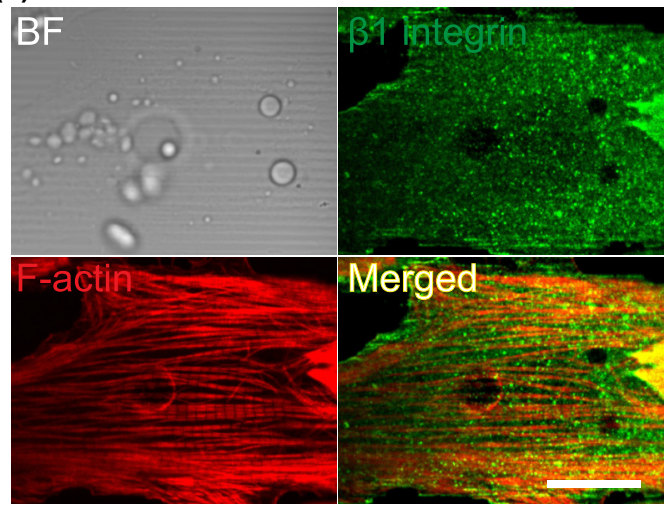

(b)

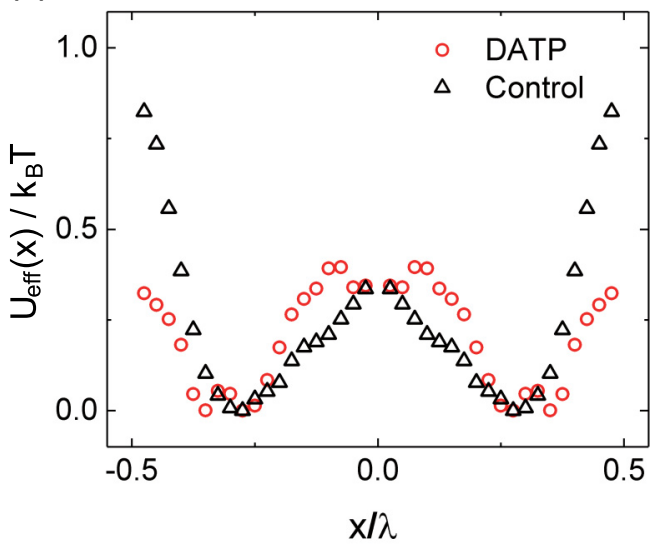

FIG. 4. Effects of ATP depletion. (a) Comparison of bright-field (BF) and costained fluorescent images of a muscle cell cultured on a $2-\mu \mathrm{m}$-microgroove-patterned substrate with the ATP depletion (DATP) treatment. The fluorescent images, which are obtained by averaging the vertical $z$-sections of the basal membrane of $\sim 1 \mu \mathrm{m}$ thickness, show the spatial distribution of $\beta_{1}$-integrins (green), cortical F-actin (red), and their superposition (merged) on the basal membrane. After ATP depletion, the stripe pattern of $\beta_{1}$-integrins, which was observed for untreated control cells, becomes invisible and their fluorescent images reveal a diffusive ground-glass pattern. Under a prolonged DATP treatment $(>1 \mathrm{~h})$, the muscle cells begin to gradually detach from the microgroove-patterned substrate, indicating a full loss of anchored integrins in the cell membrane. The scale bar is $20 \mu \mathrm{m}$. (b) Measured effective potential $U_{\text {eff }}(x)$ as a function of the normalized position $x / \lambda$ across a single microgroove with $\lambda=2 \mu \mathrm{m}$. Here $x / \lambda=0$ is set at the bottom of the microgroove. The comparison between the DATP-treated cells (red circles) and untreated control cells (black triangles) is made using the same batch of cells cultured on a 2- $\mu$ m-microgroove-patterned substrate under the same conditions, except for the use of different culture media, one with the DATP drug added and the other without the DATP drug.

the interface between the cell membrane and the ridge of the microgrooves, individual QDs moving away from the ridge region will undergo a small vertical deviation from the focal plane, which gives rise to a slight decrease in the fluorescent intensity. The relative fluorescent intensity change of the QDs thus provides a measure of the actual shape modulation of the cell membrane. Supplementary Fig. S2 [41] shows a comparison of the normalized fluorescent intensity profiles of the QD-labeled AChRs across a 2- $\mu \mathrm{m}$-microgroove for the Matrigel treated (red triangles) and untreated (black circles) cells. It is seen that the cell membrane sags slightly with an approximately $15 \%$ height variation across the microgroove and is not highly curved. The height variation for the Matrigel treated cells remains the same as that for the untreated cells.

Figure 3(c) shows the normalized fluorescent intensity profile $I(x)$ of the anchored integrins without the Matrigel treatment. By assuming $n(x) \simeq \beta\langle I(x)\rangle$, with $\langle I(x)\rangle$ being the mean value of $I(x)$ (red solid line) and $\beta$ being a proportional constant, we obtain the spatial distribution of anchored integrins $n(x)$ across a single microgroove. Without this concentration gradient, the measured effective potential $U_{\text {eff }}(x)$ for cells with the Matrigel treatment is flattened out, as shown in Fig. 3(d). Similarly, the spatial variations of the measured diffusion coefficient $D_{x}(x)$ and drift velocity $V_{x}(x)$ all disappear once the distribution of anchored integrins is randomized [see Figs. 3(e) and 3(f)]. Because the large effects of the Matrigel treatment shown in Fig. 3 are obtained when the membrane shape is kept the same as the untreated cells, they clearly demonstrate that the effective trapping potential $U_{\text {eff }}(x)$ and its effects on the AChR's motion are indeed caused by the nonuniform distribution of anchored integrins but not by some geometric effects of membrane shape, such as membrane curvature or any direct interactions of AChRs with either the curved substrate or the cortical actin network.

Because of the anisotropy of the microgroove pattern, the cortical F-actin filaments are found to be aligned along the trough of the microgrooves (see supplementary Fig. S1 [41]). The local accumulation of F-actin filaments allows other transmembrane proteins to anchor in the trough region, which gives rise to a small bump in the measured $U_{\text {eff }}(x)$ around $x / \lambda \simeq 0$ [see Figs. $1(\mathrm{~d})$ and $3(\mathrm{~d})$ ]. A similar effect is also observed in the measured $U_{\text {eff }}(x)$ for cells with the Matrigel treatment. As shown in Figs. 3(d)-3(f) (red circles), the effect of this local accumulation of F-actin filaments is small and can be treated as a background contribution, which can be subtracted out from the actual signal (see the discussions on Fig. 5 below).

Second, cellular ATP is needed to keep the integrins at the anchoring state against diffusion and fluctuations from the surrounding molecules. Recent studies have shown that contractile forces from the cytoskeletal network are required to activate integrins from their low-affinity conformation to high-affinity conformation for the adhesion/anchoring of integrins to the extracellular matrix $[14,42]$. The generation of contractile forces by motor proteins requires an energy input from ATP. In our experiment, we find that depletion of the ATP in live muscle cells by a 1:1 mixture of 2-deoxy-D-glucose and sodium azide [15,43] significantly reduces the number of anchored integrins on the ridge of microgrooves (see Sec. IV C 1 below for more experimental details). After ATP-depletion, the stripe pattern of $\beta_{1}$-integrins becomes invisible and their fluorescent images reveal a diffusive ground-glass pattern, as shown in Fig. 4(a). Meanwhile, the resulting effective potential $U_{\text {eff }}(x)$ is leveled off with the 
barrier height $E_{b}$ reduced by $\sim 60 \%$, as shown in Fig. 4 (b). The loss of anchored integrins after ATP-depletion is further evidenced by a nearly fivefold increase of the diffusion coefficient $D_{x}(x)$ of AChRs compared to the control case, as shown in supplementary Fig. S3 [41].

While the fluorescent image of integrins appears uniform after ATP-depletion, there must be some integrins anchored on the ridge of microgrooves, as the muscle cell itself still remains attached to the substrate during the experiment. As a result, the F-actin network is still aligned along the trough of the microgrooves [see Fig. 4(a)], and the effective potential $U_{\text {eff }}(x)$ reveals a weak undulation pattern [see Fig. 4(b)]. Compared to the significant reduction of the spatial undulation amplitude of anchored integrins by ATP-depletion, the Matrigel treatment discussed above results in an enhanced uniform distribution of anchored integrins across the whole plasma membrane, so that the short-time diffusion coefficient $D_{x}(x)$ of AChRs is reduced significantly and becomes a constant across the microgroove, as shown in Fig. 3(e). Although ATP depletion may have other effects on living cells, Fig. 4 shows the direct experimental observation of the effects of ATP depletion on anchored integrins. The experimental findings suggest that living cells may use the energy input from ATP to actively regulate the spatial distribution of anchored proteins and produce a free-energy landscape for molecular transport in the mesoscale.

To check whether the above experimental findings are influenced by active agitations from the actin cortex $[4,6]$, we repeat the same measurements as those shown in Fig. 2 for the cells that are treated by blebbistatin to inhibit the activity of myosin II motors. Myosin II is a nonprocessive motor protein that generates active contractions in the cortical F-actin network upon ATP hydrolysis. In the blebbistatin treatment, we follow the standard protocol [43] of adding $10 \mu \mathrm{M}$ blebbistatin to the cell culture medium for $30 \mathrm{~min}$. Supplementary Fig. S4 [41] shows a comparison of the measured effective potential $U_{\text {eff }}(x)$, short-time diffusion coefficients $D_{x}(x)$ and $D_{y}(x)$, and drift velocities $V_{x}(x)$ and $V_{y}(x)$ across the microgroove for the blebbistatin-treated cells (red symbols) and untreated cells (black symbols). It is seen that the blebbistatin treatment does not make any significant change to the measured profiles of $U_{\text {eff }}(x), D_{x}(x), D_{y}(x), V_{x}(x)$, and $V_{y}(x)$. This result thus demonstrates that the active agitations from the actin cortex do not play a major role in the measurements discussed above.

\section{FURTHER ANALYSIS AND DISCUSSIONS}

\section{A. Comparison with theory}

To quantitatively describe the motion of AChRs in the potential field $U(x)$ generated by anchored integrins, we consider the PDF $H\left(x, t ; x_{0}, t_{0}\right)[\equiv H(x, t)$ for short of finding a mobile AChR at the position $x$ and time $t$, whose initial spacetime position is $x_{0}$ and $t_{0}$. The 1D Smoluchowski equation for $H(x, t)$ reads [44]

$$
\frac{\partial H(x, t)}{\partial t}=\frac{\partial}{\partial x}\left[\frac{d U(x)}{d x} \frac{H(x, t)}{\xi(x)}+D_{x}(x) \frac{\partial H(x, t)}{\partial x}\right],
$$

where $\xi(x)$ and $D_{x}(x)$ are, respectively, the friction and diffusion coefficients of mobile AChRs; both are functions of $x$.
Note that Eq. (1) is quite general and holds even when $\xi(x)$ and $D(x)$ do not obey the Stokes-Einstein relation. From the zero-flux steady-state solution, $H(x)=H(0) \exp \left\{-\left[U_{\text {eff }}(x)-\right.\right.$ $\left.\left.U_{\text {eff }}(0)\right]\right\}$, one obtains an effective potential $U_{\text {eff }}(x)$, which is equal to the potential field $U(x)$ only when the AChR dynamics is passive.

To obtain the potential field $U(x)$ produced by fixed obstacles (anchored integrins), a one-dimensional lattice model for the passive diffusion of AChRs in the presence of the obstacles (see Appendix 1 for more details) is applied to derive another Smoluchowski equation,

$$
\begin{aligned}
\frac{\partial H(x, t)}{\partial t}= & D_{0} \frac{\partial}{\partial x}\left[\left(\frac{d c(x) / d x}{1+\alpha n(x)}-\frac{\alpha[1-c(x)] d n(x) / d x}{[1+\alpha n(x)]^{2}}\right)\right. \\
& \left.\times H(x, t)+\frac{1-c(x)}{1+\alpha n(x)} \frac{\partial H(x, t)}{\partial x}\right],
\end{aligned}
$$

where $c(x)$ is the probability that the site $x$ cannot be crossed due to the obstacles and $D_{0} \equiv(d x)^{2} /(2 d t)$. The factor $1+$ $\alpha n(x)$ is introduced to account for interactions between the mobile AChRs and anchored integrins, where $\alpha$ is a proportional constant. With the equilibrium solution $H(x)$ of Eq. (2) and the Boltzmann distribution $U(x)=-k_{B} T \ln H(x)$, we find

$$
U(x) \simeq-k_{B} T \ln \{[1-n(x)][1+\alpha n(x)]\},
$$

where we have assumed that $c(x)$ is proportional to the concentration $n(x)$ of anchored integrins, i.e., $c(x) \simeq n(x)$. For small values of $n(x)$ and assuming $\alpha=0$, we have $U(x) \simeq$ $n(x) k_{B} T$, as discussed above.

By moment expansion of the time-dependent PDF $H(x, t)$ and comparing Eq. (1) with Eq. (2) (see Appendix 2 for more details), we obtain an analytic expression of three dynamic properties of the AChRs that can be tested directly by experiment. The first prediction is the short-time diffusion coefficient

$$
D_{x}(x) \simeq D_{0} \frac{1-n(x)}{1+\alpha n(x)} \equiv D_{l}(x),
$$

where the notation $D_{l}(x)$ is introduced to indicate that this expression is obtained based on the passive lattice model.

The second prediction is about the drift velocity,

$$
V_{x}(x) \simeq \begin{cases}\frac{-D_{x}(x)}{k_{B} T} \frac{d U(x)}{d x}+\frac{d D_{x}(x)}{d x} & \text { from Eq. (1), } \\ -2 D_{0} \frac{d n(x) / d x}{1+\alpha n(x)} \equiv V_{l}(x) & \text { from Eq. (2). }\end{cases}
$$

Again, the notation $V_{l}(x)$ is used to indicate that this expression is obtained based on the passive lattice model. Equation (5) suggests that the AChRs will move toward a less populated region of anchored integrins, and their velocity is proportional to the concentration gradient of anchored integrins.

The third prediction is an analytic relationship between the dynamic properties of the AChRs and the effective potential $U_{\text {eff }}(x)$ :

$$
U_{\mathrm{eff}}(x)=U_{0}-k_{B} T \int_{0}^{x} \frac{V_{x}(u)-d D_{x}(u) / d u}{D_{x}(u)} d u,
$$

where $U_{0}$ is an integration constant.

To compare with the theory, we numerically calculate the potential $U_{\text {eff }}(x)$ using Eq. (6) with the measured $D_{x}(x)$ and 


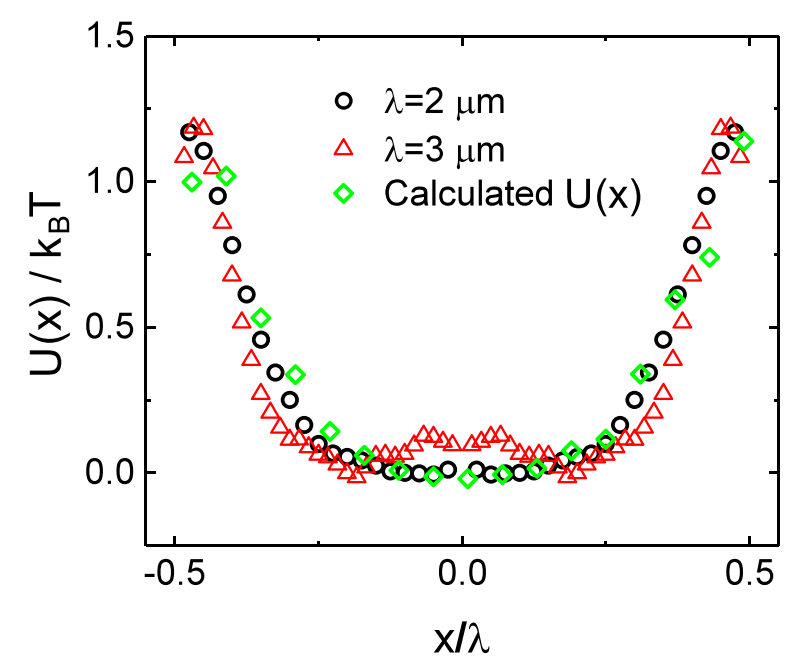

FIG. 5. Potential field $U(x)$ generated by anchored integrins alone. The potential $U(x)$ (black circles) is obtained by subtracting the red circles from the black triangles in Fig. 3(d). The calculated $U(x)$ (green diamonds) is obtained by using Eq. (3) with $n(x)=$ $\beta\langle I(x)\rangle$, where $\langle I(x)\rangle$ is the mean fluorescent intensity profile of $\beta_{1}$-integrins, as shown in Fig. 3(c). The best fit is obtained with $\alpha=0.55 \pm 0.05$ and $\beta=0.95 \pm 0.05$. The red triangles show the potential $U(x)$ obtained from muscle cells cultured on a $3-\mu \mathrm{m}-$ microgroove-patterned substrate.

$V_{x}(x)$ shown in Figs. 3(e) and 3(f), and the final result is shown by the green diamonds in Fig. 3(d). The calculated $U_{\text {eff }}(x)$ agrees well with the experimental data (black triangles) without any adjustable parameter. This result demonstrates that Eq. (6) is a generic relationship, which is valid even when the local (nonuniform) distribution of anchored proteins involves multiple species.

To obtain the potential field $U(x)$ resulting solely from the anchored integrins, we treat the red circles in Fig. 3(d) as the background contribution from other anchored proteins in the region and subtract them from the measured $U_{\text {eff }}(x)$ (black triangles) in Fig. 3(d). Figure 5 shows a comparison between this corrected potential $U(x)$ (black circles) and the calculated $U(x)$ (green diamonds) using Eq. (3) with $n(x)=$ $\beta\langle I(x)\rangle$, where $\langle I(x)\rangle$ is the mean fluorescent intensity profile of $\beta_{1}$-integrins, as shown in Fig. 3(c). A good agreement is observed between the measured $U(x)$ and the theoretical prediction based on the passive lattice model. The best fit in Fig. 5 is obtained with $\alpha=0.55>0$, suggesting that there is an attractive interaction between the mobile AChRs and anchored integrins [45]. From the above results, we conclude that the motion of AChRs is adequately described by the equilibrium dynamics. This conclusion is further supported by the measured time correlation function, $C(t)=\langle\delta x(t) \delta x(0)\rangle$, of the AChR movement in the direction normal to the microgroove. Here $\delta x(t)=x(t)-\langle x(t)\rangle$ is the deviation of the AChR's position $x(t)$ from its mean value $\langle x(t)\rangle$. As shown in supplementary Fig. S5 [41], the measured $C(t)$ reveals a time-reversal symmetry (within the experimental uncertainties), which is a signature for fluctuations at equilibrium [44]. Because of the effects of molecular motors and ATP-driven polymerization forces, this equilibrium may not be an exact thermal equilibrium but an effective equilibrium with an elevated temperature.

To account for the background contributions from other anchored proteins to $D_{x}(x)$, we rewrite Eq. (4) as

$$
\frac{D_{x}(x)-D_{\min }}{D_{\max }-D_{\min }} \simeq \frac{1-n(x)}{1+\alpha n(x)},
$$

which has the same form as Eq. (4) but with an added baseline $D_{\min }$ and $D_{0}=D_{\max }-D_{\min }$. The green diamonds in Fig. 3(e) show the calculated $D_{x}(x)$ using Eq. (7) with similar fitting parameters to those for $U(x)$ in Fig. 5. The calculated $D_{x}(x)$ agrees well with the experimental result. The obtained $D_{\max }=0.058 \mu \mathrm{m}^{2} / \mathrm{s}$ and $D_{\min }=0.028 \mu \mathrm{m}^{2} / \mathrm{s}$ are close to the expected values shown in Fig. 3(e).

The blue dashed line in Fig. 3(f) shows the calculated $V_{x}(x)$ [or $V_{l}(x)$ ] using the second equality in Eq. (5) with the same values of $D_{0}, \alpha$, and $\beta$ as those for $D_{x}(x)$. This time the calculated $V_{x}(x)$ does not fit the data well, because the measured $V_{x}(x)$ is sensitive to small concentration gradients of all other anchored proteins, which are not included in the equation and cannot be simply corrected by adding a constant baseline. Nonetheless, we find that the first equality in Eq. (5) (green diamonds) fits the data well. Note that the first equality in Eq. (5) is just the derivative form of Eq. (6), and thus the fitting result shown in Fig. 3(f) further confirms that Eq. (6) is an intrinsic relationship among the three measured quantities $U_{\text {eff }}(x), D_{x}(x)$, and $V_{x}(x)$. The fitting results shown in Figs. 3 and 5 demonstrate that our model on the directed motion of membrane proteins under the entropy-driven potential field generated by anchored proteins captures the essential physics.

We also conduct comparative tracking measurements of AChRs for muscle cells cultured on a $3-\mu \mathrm{m}$-microgroovepatterned substrate with and without the Matrigel treatment. Supplementary Fig. S6 [41] shows the experimental results, which are presented in the same way as Fig. 3. It is found that the three measured quantities $U_{\text {eff }}(x), D_{x}(x)$, and $V_{x}(x)$ for the $3-\mu \mathrm{m}$-microgrooves behave the same as those for the $2-\mu \mathrm{m}$-microgrooves. For example, the red triangles in Fig. 5 show the corrected potential $U(x)$ for the $3-\mu \mathrm{m}$ microgrooves, which overlaps with the measured $U(x)$ for the 2- $\mu \mathrm{m}$-microgrooves once the position $x$ across the microgroove is normalized by the microgroove spacing $\lambda$. It is seen that the barrier height $E_{b}$ remains approximately the same when $\lambda$ is changed from 2 to $3 \mu \mathrm{m}$. While the groove height is kept unchanged in the experiment, we believe it is not a sensitive parameter to change $E_{b}$ either, so long as the groove height remains tall enough so that the portion of the cell membrane over the microgrooves can be kept suspended. As shown in Eq. (3), the value of $E_{b}$ depends sensitively on the concentration variation $n(x)$ of anchored integrins, which is determined primarily by the available integrins in the cell membrane and the local contact area between the cell membrane and the ridge of parallel microgrooves. The latter is not very sensitive to the geometrical parameters of the microgrooves, such as their spacing and height, used in the experiment.

A noticeable difference between the two cases is that the local alignment and accumulation of F-actin in the wider trough region of the 3- $\mu \mathrm{m}$-microgrooves (see supplementary Fig. S6 [41]) have a larger effect compared with that for 
the 2- $\mu \mathrm{m}$-microgrooves. As a result, the corrected potential $U(x)$ for the 3- $\mu \mathrm{m}$-microgrooves still exhibits a small residual bump near $x / \lambda \simeq 0$, because of the limited statistics of the measured $U_{\text {eff }}(x)$ for cells with the Matrigel treatment [see Fig. S6(d) [41]]. The effect of the local accumulation of extra anchored proteins is also reflected in the measured $D_{x}(x)$ with a small dent [see Fig. S6(e) [41]] and a small drift velocity $V_{x}(x)$ [see Fig. S6(f) [41]] in the trough region.

To illustrate the confining effect of the resulting potential $U(x)$, we measure the residence time $t_{R}$ of the AChRs diffusing inside the potential trap induced by the 2$\mu \mathrm{m}$-microgroove-patterned substrate. The obtained histogram $H\left(t_{R}\right)$ of the residence time $t_{R}$ (see supplementary Fig. S7 [41]) is found to be well described by a simple exponential function, $H\left(t_{R}\right) \sim \exp \left(-t_{R} /\left\langle t_{R}\right\rangle\right)$. This result suggests that the AChR hopping events between different microgrooves occur randomly in time and can be described by a Poisson process. The obtained mean residence time $\left\langle t_{R}\right\rangle \simeq 42 \mathrm{~s}$ is in agreement with the crossover time $\tau_{H}$ shown in Fig. 2(a) (blue circles), above which the measured $\operatorname{MSD}\left\langle\Delta x^{2}(\tau)\right\rangle$ enters the longtime diffusion regime. In this regime, the AChRs have hopped over enough energy barriers so that their trajectories are fully randomized.

\section{B. Biological implications}

The above results suggest that anchored proteins in the membrane are not simply passive random obstacles for mobile proteins, they may also play an active role in generating an effective potential landscape to guide molecular motion in the mesoscale. This finding offers a transport mechanism that has important biological implications.

First, anchored proteins can help to build molecular traps, such as those "hot spots" observed on the plasma membrane of various cell types [46-48], which facilitate the compartmentalization of receptors and downstream signaling molecules for more efficient signaling. To demonstrate this effect, we fabricate identical cylindrical holes (microwells) of diameter $d=2 \mu \mathrm{m}$ and depth $=1 \mu \mathrm{m}$ arranged on a hexagonal lattice of $2.5 \mu \mathrm{m}$ in lattice constant. The 2D microwell-patterned surface is made on a thin layer of PDMS using an approach similar to that for microgroove patterning (see Secs. IV A and IV B below for more experimental details). Figure 6(a) reveals that the $\beta_{1}$-integrins are strongly enriched at the ridge of microwells and form a continuous and periodic network on the basal membrane, which has a similar pattern to that of the substrate. For the muscle cells cultured on a flat glass coverslip, on the other hand, the $\beta_{1}$-integrins are found to be evenly distributed across the basal membrane without any ordered pattern. Figure 6 thus suggests that the basal membrane is freely suspended over the microwells, and the $\beta_{1}$-integrins are predominantly anchored on the ridge of microwells.

From the AChR trajectories, we obtain the PDF $H(x, y)$ of finding an AChR at location $(x, y)$ and corresponding potential $U_{\text {eff }}(x, y) / k_{B} T=-\ln H(x, y)$. Figures 6(b) and 6(c) show, respectively, the $1 \mathrm{D}$ and $3 \mathrm{D}$ plots of the obtained potential trap with the trap boundary uniquely defined by the ridge of the microwells where the integrins reside. The barrier height $E_{b}$ of the trap is $E_{b} \simeq 2 k_{B} T$. Over this potential landscape,
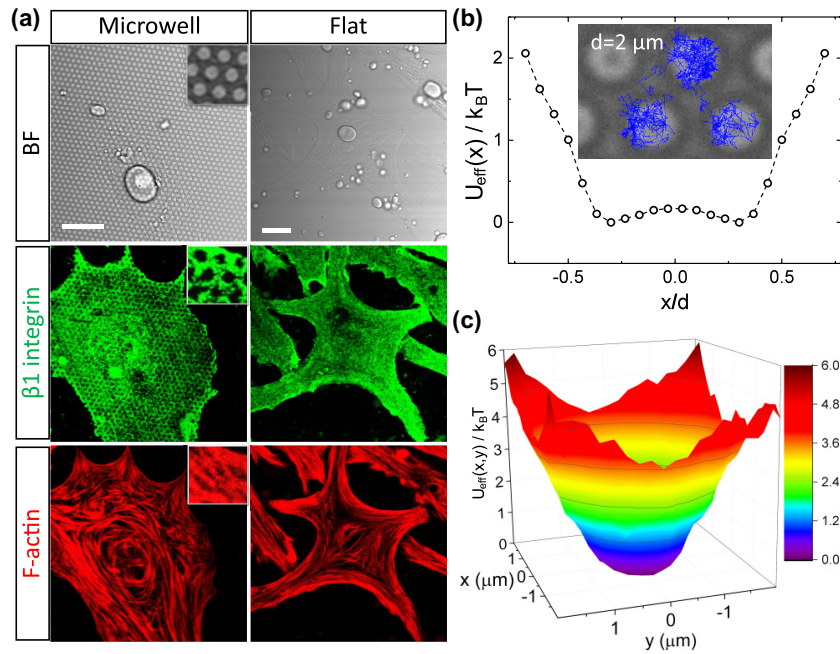

FIG. 6. Microwell-patterned substrate induces a periodic array of 2D potential traps for AChRs. (a) Comparison of bright-field (BF) and costained fluorescent images of a muscle cell cultured on a $2-\mu \mathrm{m}$-microwell-patterned substrate (left panel) and on a flat substrate (right panel). The fluorescent images, $z$-sections-averaged for the patterned substrate, show the spatial distribution of $\beta_{1}$-integrins (green) and cortical F-actin (red) on the basal membrane. The insets show a magnified view of a portion of the images. Scale bars are $20 \mu \mathrm{m}$. (b) $1 \mathrm{D}$ plot of the measured trapping potential $U_{\text {eff }}(x)$ for mobile AChRs across the microwell diameter $x / d$ with $d=2 \mu \mathrm{m}$. Here $x / d=0$ is set at the microwell center. The inset shows a representative AChR trajectory (blue) with 1500 time steps (300 s) over the bright-field microwell pattern. (c) 3D plot of the measured trapping potential $U_{\text {eff }}(x, y)$ for mobile AChRs. Approximately 8000 AChR trajectories are used to obtain $U_{\text {eff }}(x, y)$.

the AChR trajectories exhibit a short-term confined diffusion within a trap and an occasional long-term hopping between traps [inset of Fig. 6(b)]. The value of $E_{b}$ remains approximately the same when the diameter of the microwells is changed to $d=3 \mu \mathrm{m}$ (see supplementary Fig. S8 [41]).

Molecular traps or "hot spots" with similar values of $E_{b}$ were also reported for physiologically relevant systems. For example, at the inhibitory synapse, glycine neurotransmitter receptors were found to encounter potential traps with $E_{b} \simeq$ $3 k_{B} T$, which involve integrins and scaffolding molecules gephyrin $[46,47]$. On the plasma membrane of the Chinese hamster ovary (CHO)-K1 cells and primary human umbilical vein endothelial cells, G-protein-coupled receptors were found to experience "hot spots" with $E_{b} \simeq 1 k_{B} T$, which involve actin fibers, microtubules, and clathrin-coated pits [48]. The size of these potential traps is in the range of 100-200 nm, which is much larger than the interaction range of individual proteins.

Second, a concentration gradient of anchored proteins can produce directed motion along the gradient for intracellular transport in the mesoscale. The axon initial segment (AIS) that sits between the neuron cell body and the axon is an example in which the cell adhesion molecules-neurofascins-were found to have a long-range concentration gradient up to $\sim 60 \mu \mathrm{m}$ (with a reducing population along the axon) [49]. The location and length of AIS are important for neurons to 
fine-tune their excitability and modulate their plasticity in response to activity $[12,50]$. The concentration gradient of anchored neurofascins in the AIS may provide an effective channel for directed transport of membrane proteins in the region. This hypothesis is supported by the experimental finding that the diffusion coefficient of membrane proteins increases gradually along the AIS [26]. As shown in Eq. (5), such an increase in diffusion coefficient leads to a directed drift velocity along the AIS.

The concentration gradient of anchored proteins could be utilized to build a mesoscale funnel-shaped potential landscape around a molecular trap to guide the molecular motion toward the trap. For example, during synaptogenesis of the vertebrate neuromuscular junction (NMJ), the dispersed AChRs become highly concentrated on the postsynaptic membrane of the NMJ after motor nerve innervation for a few hours [28,51]. A long-standing debate is how can an AChR move into the nerve-muscle contact region? The diffusiontrap hypothesis $[52,53]$ assumes that the AChRs diffuse into the junctional region and are trapped at the site of nerve contact. Because the contact area $\delta s$ is much smaller than the open area $S$ available for free diffusion of AChRs, the probability for an AChR to get trapped in the contact area by sheer luck, $p \sim \delta s / S$, is very small $(<0.1 \%)$ [51]. The probability for $N$ AChRs to get trapped in the same area, $p^{N}$, is even smaller. Thus, free diffusion in open space, such as a $2 \mathrm{D}$ plasma membrane, is not very efficient for molecular transport to a targeted location. Directed motion by a mesoscale funnel-shaped potential landscape could provide an answer.

Finally, other spatial arrangements of anchored proteins, such as protein scaffolds [51,54] and condensates [55,56], may also help to build a local potential landscape to enhance the protein-protein interactions by increasing their interaction range. To fulfill their biological functions, the anchored protein networks or clusters need to be stable over a long enough time against diffusion and fluctuations from the surrounding mobile molecules. This lock in place (or "brake" [2]), however, does not need to be permanent and can be modified over a longer timescale. In this way, molecular transport in the mesoscale can be actively regulated in living cells via the anchoring dynamics with either extracellular or intracellular matrices, which requires a constant input of energy from the ATP hydrolysis.

In this work, we use an ordered array of anchored integrins to generate a free-energy landscape to guide the motion of membrane proteins and trap them in space. Like many other proteins inside a living cell, the membrane proteins that we refer to here are nonmotor proteins, which undergo a stochastic random motion when no external potential is applied. The free-energy landscape induced by anchored integrins provides a biased potential equal to or larger than $1 k_{B} T$, so that a single protein molecule can overcome its random motion and move to a location with a lower free-energy. What matters here is the potential difference, which sets the direction of molecular motion, rather than the actual value of the force involved, which only determines how fast the protein molecule can reach its destination. This is because the mobile proteins of interest are usually abundant in the cell membrane, and the typical timescales involved in intracellular transport are in (i) Spin coating

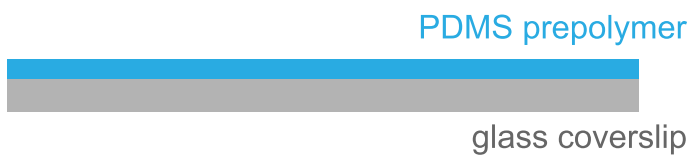

(ii) Molding

silicon mold with a microgroove or microwell pattern

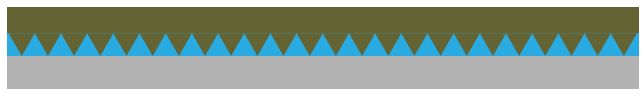

(iii) Thermal curing

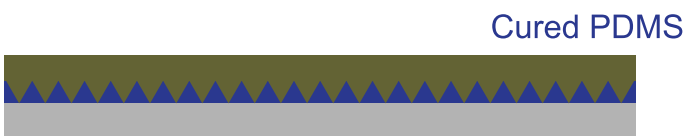

(iv) Peeling off

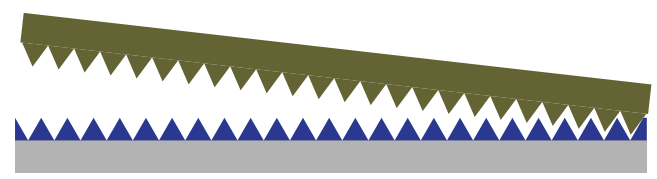

(v) ECL coating

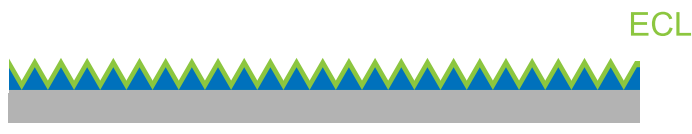

FIG. 7. A schematic illustration of the fabrication processes of the microwell- and microgroove-patterned PDMS substrates on a glass coverslip.

hours and even days [57]. This time is adequate for most protein transport processes, and thus they are not diffusionlimited [53].

While the resulting landscape is determined primarily by physical interactions and thus is uniformly applicable to all the membrane proteins, this coarse-grained approach can be readily expanded to construct a potential trap for a specific type of protein by using a mixture of anchored proteins with different chemical interactions with the targeted proteins. In fact, such a chemically specific trap for glycine neurotransmitter receptors at inhibitory synapses has been identified and characterized by Masson et al. [47]. The trap involves integrins and gephyrin scaffolds. Evidently, further investigations are needed to test the hypotheses and predictions as discussed above.

\section{MATERIALS AND METHODS}

\section{A. Fabrication of microwell- and microgroove-patterned PDMS substrates on a glass coverslip}

The technique of micropatterning was used to construct a patterned surface with either identical cylindrical holes (microwells) or parallel microgrooves. Figure 7 illustrates the main fabrication procedures. (i) A thin layer of poly(dimethylsiloxane) (PDMS) prepolymer [a 10:1 mixture (by $w / w$ ) of the base material and curing agent] was 

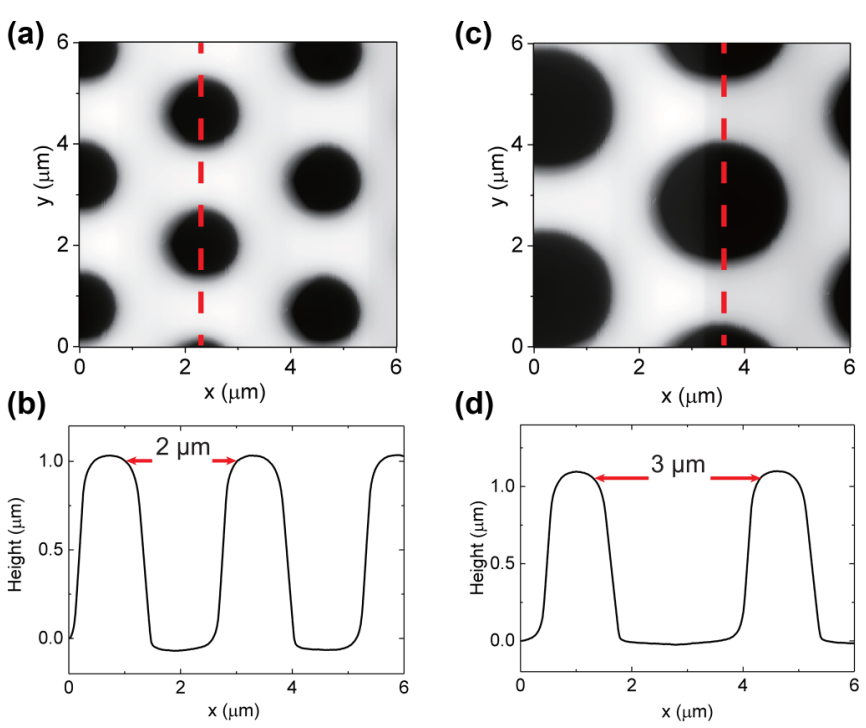

FIG. 8. (a) AFM topographic image of the top surface of the 2$\mu$ m-microwell patterned PDMS substrate in air. (b) Cross-sectional view of the measured height image of the PDMS top surface. The height profile is obtained along the vertical direction as indicated by the red dashed line shown in (a). (c) AFM topographic image of the top surface of the 3- $\mu \mathrm{m}$-microwell patterned PDMS substrate in air. (d) Cross-sectional view of the measured height image of the PDMS top surface. The height profile is obtained along the vertical direction as indicated by the red dashed line shown in (c).

spin-coated on a precleaned glass coverslip at $4000 \mathrm{rpm}$ for $10 \mathrm{~min}$. (ii) A silanized silicon mold [vapor-coated with dichlorodimethylsilane (Sigma)] carrying either the microwell or microgroove feature was brought in close contact with the PDMS-coated glass coverslip. The silicon molds with the microwell and microgroove patterns were made, respectively, by deep-reactive ion etching and anisotropic wet etching in a tetramethyl ammonium hydroxide (TMAH) solution, as described previously in Ref. [58]. (iii) The silicon-PDMSglass-coverslip sandwich was heated at $100{ }^{\circ} \mathrm{C}$ for $1 \mathrm{~h}$. (iv) After the thermal curing, the micropatterned PDMS substrate was peeled off from the silicon mold. (v) The micropatterned glass coverslip was then cleaned, dried, and subsequently treated by $\mathrm{O}_{2}$ plasma for 1 min before being coated with a thin layer of ECL cell attachment matrix [containing primarily laminin, collagen IV, and entactin with a 1:200 dilution by the culture medium (Upstate Co.) at $37{ }^{\circ} \mathrm{C}$ for $1 \mathrm{~h}$ ]. The glass coverslip was placed in a $35-\mathrm{mm}$ polystyrene tissue-culture dish and further rinsed with phosphate buffered saline (PBS) for three times before cell seeding.

\section{B. Atomic force microscopy characterization}

An atomic force microscope (MFP-3D, Asylum Research) was used to examine the surface profile of the microwelland microgroove-patterned PDMS substrates. Figure 8 shows the atomic force microscopy (AFM) topographic images of the top surface of two microwell-patterned PDMS substrates in air. From the measured height profile of the microwell patterns, we find that the resulting microwell patterns are smooth and uniform in size and depth. The microwells shown
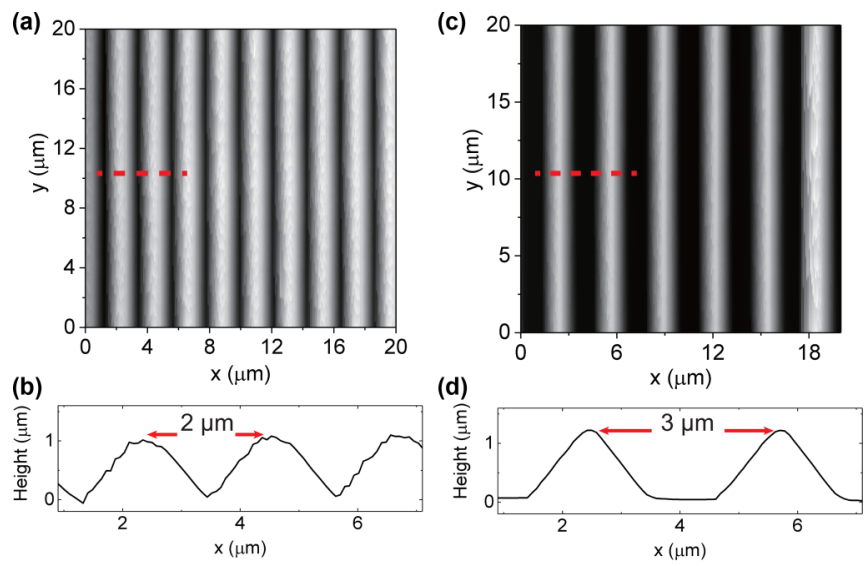

FIG. 9. (a) AFM topographic image of the top surface of the 2- $\mu$ m-microgroove patterned PDMS substrate in air. (b) Crosssectional view of the measured height image of the PDMS top surface. The height profile is obtained along the horizontal direction as indicated by the red dashed line shown in (a). (c) AFM topographic image of the top surface of the 3- $\mu \mathrm{m}$-microgroove patterned PDMS substrate in air. (b) Cross-sectional view of the measured height image of the PDMS top surface. The height profile is obtained along the horizontal direction as indicated by the red dashed line shown in (c).

in Fig. 8(a) have a uniform diameter of $d=2 \mu \mathrm{m}$, and their center-to-center separation is $2.5 \mu \mathrm{m}$. The microwells shown in Fig. 8(c) have a uniform diameter of $d=3 \mu \mathrm{m}$, and their center-to-center separation is $3.5 \mu \mathrm{m}$. The two kinds of microwells have the same depth of $1 \mu \mathrm{m}$.

Figure 9 shows the AFM topographic images of the top surface of two microgroove-patterned PDMS substrates in air. From the measured height profile of the microgroove patterns, we find that the resulting microgroove patterns are smooth and uniform and have a triangle-shaped cross-section. The microgrooves shown in Fig. 9(a) have a uniform spacing of $\lambda=$ $2 \mu \mathrm{m}$. The microgrooves shown in Fig. 9(c) have a uniform spacing of $\lambda=3 \mu \mathrm{m}$. The two kinds of microgrooves have the same height of $1 \mu \mathrm{m}$.

\section{Preparation of biological samples}

The experimental procedures used in this work, such as cell culture, drug treatments, quantum dot (QD) labeling, immunostaining, and optical and confocal imaging, have been described in detail in previous publications [13,15], and here we only mention some key features.

\section{Cell culture and drug treatments}

The AChR is a cation-selective, ligand-gated ion channel and consists of five subunits with diameter $\sim 7 \mathrm{~nm}$. It is an integral membrane protein that responds to the binding of acetylcholine, which is a neurotransmitter. The AChR spans the membrane of muscle cells with most of its mass in the extracellular space [59]. Muscle cells were obtained from dissection of fertilized embryos of Xenopus laevis (or African clawed frog) at the stages $20-22$, as described previously in Ref. [60]. The dissected muscle cells were then seeded on flat or micropatterned glass coverslips coated with a thin layer of 
an ECL cell attachment matrix and raised in a culture medium composed of $88 \%$ Steinberg's solution, $10 \%$ L-15 medium (Leibovitz Co.), $1 \%$ fetal bovine serum, and $1 \%$ gentamicin $\left(50 \mathrm{mg} / \mathrm{mL}\right.$, Sigma-Aldrich) and maintained at $23^{\circ} \mathrm{C}$.

To alter the spatial distribution of $\beta_{1}$-integrins in the plasma membrane of live muscle cells cultured on the microgroove-patterned substrate, the culture medium was supplemented with the growth-factor-reduced Matrigel (Corning, no. 354230) at a concentration of $0.45 \mathrm{mg} / \mathrm{mL}$. The Matrigel comprises primarily soluble extracellular matrix (ECM) proteins, including laminin, collagen IV, and entactin, and it was applied to 2-day-old muscle cells following their attachment and spreading on the microgroove-patterned substrate. Single-particle tracking of AChRs was performed 1 day after the addition of Matrigel. A control group was prepared using the same batch of cells and culture medium without Matrigel added.

To reduce the intracellular ATP (adenosine triphosphate) level in living muscle cells, the cells were treated with a 1:1 mixture of 2-deoxy-D-glucose (30 mM, Sigma-Aldrich) and sodium azide (30 mM, Sigma-Aldrich) in the PBS buffer for $1 \mathrm{~h}$ [43] and then imaged in PBS for tracking experiments. Cells in the control group were incubated in PBS for tracking experiments.

\section{Immunostaining and confocal microscopy}

The entire staining process was performed at room temperature. Briefly, the muscle cells were fixed with $4 \%$ paraformaldehyde in PBS (for $10 \mathrm{~min}$ ) and subsequently permeabilized with $0.2 \mathrm{M} \mathrm{NH}_{4} \mathrm{CL} / \mathrm{PBS}$ containing $0.2 \%$ Triton $\mathrm{X}-100$ (for $10 \mathrm{~min}$ ), and then blocked with $4 \%$ bovine serum albumin (Sigma-Aldrich) in PBS (for $2 \mathrm{~h}$ ). To measure the spatial distribution of $\mathrm{F}$-actin and $\beta_{1}$-integrins simultaneously, the cells were first incubated with mouse monoclonal antibodies against the Xenopus $\beta_{1}$ integrin subunit $(8 \mathrm{C} 8,1: 50$ dilution, DSHB) for $1 \mathrm{~h}$ [61]. After washing with PBS (5 min each for three times), the cells were incubated with Alexa Fluor 488 conjugated goat antimouse IgG (1:200 dilution, Abcam) and phalloidin-TRITC $(0.2 \mu \mathrm{g} / \mathrm{mL}$, Sigma-Aldrich) for $1 \mathrm{~h}$. Finally, the cell sample was washed with PBS (5 min each for three times) and mounted onto a microscope slide with Citifluor-AF-1 (Ted Pella), and then examined under a confocal microscope (LSM710, Zeiss).

The fluorescent images for muscle cells cultured on the patterned substrate were collected over a stack of vertical $z$-sections of the basal membrane of $\sim 1 \mu \mathrm{m}$ thickness. The final fluorescent images shown in the main text are the $z$ averaged images of the whole basal membrane by using the ImageJ software (NIH). To obtain the normalized mean intensity profile $\langle I(x)\rangle$ associated with the $\beta_{1}$-integrins over a single microgroove period, the relative fluorescent intensity $i(x)$ over different microgrooves for a single cell was first normalized to become $i_{n}(x)$, which is defined as $i_{n}(x)=[i(x)-$ $\left.i_{\min }(x)\right] /\left[i_{\max }(x)-i_{\min }(x)\right]$, where $i_{\min }(x)$ and $i_{\max }(x)$ are, respectively, the minimal and maximal intensities obtained for the cell. The intensity profile $i_{n}(x)$ was then overlayed into a single microgroove period to obtain $I(x)$ for each cell. Figure 3(c) shows the resulting $I(x)$ from different cells, and $\langle I(x)\rangle$ is the averaged intensity profile over all the data points.

\section{QD labeling and optical imaging}

To track the AChRs on a live muscle cell membrane, individual AChRs are labeled by bright and photostable fluorescent QDs [62,63]. This is achieved by first labeling the AChRs with biotinylated $\alpha$-bungarotoxin (biotin-BTX, 0.5 $\mathrm{nM}$, Invitrogen Co.) for $10 \mathrm{~min}$. The cells are then washed with the culture medium three times ( 5 min each). After repeated washing to remove unbounded biotin-BTX, $2.5 \mathrm{nM}$ streptavidin-conjugated Qdot 655 solution (Thermo Fisher Scientific) is added to the cells for $10 \mathrm{~min}$, after which the cells are washed with the culture medium three times ( $5 \mathrm{~min}$ each).

The live-cell sample cultured on a micropatterned or flat glass coverslip was then mounted on a coverslip holder (SC15012, Aireka Cells), which was placed on an inverted microscope (DM-IRB, Leica) with a $100 \times$ objective (NA $=1.40$ ). The motion of QDs (and hence AChRs) was viewed from below with fluorescence microscopy. Image sequences were recorded using an ultralow noise sCMOS camera (ZYLA-4.2P, Andor Technology) and streamed to the hard drive of a host computer. Image acquisition was controlled by IQ 3.1 software (Andor Technology). The QDs were excited by visible light with wavelength $400-450 \mathrm{~nm}$, and the light emitted by the QDs was selected to be in the wavelength range $645-655 \mathrm{~nm}$. A typical frame rate used in the movie recording was $5 \mathrm{fps}$ for 5 min with a spatial resolution of $2048 \times 2048$ pixels. The exposure time for each frame is $30 \mathrm{~ms}$, and a mechanical shutter was used to control the overall UV exposure time and reduce the damage to the living cells. The recorded images have 16 bits of gray scales, and each QD typically occupies 11 pixels across their diameter. The width of each pixel is $50 \mathrm{~nm}$ under our optical setup.

Fresh Xenopus muscle cells cultured for 2-3 days after dissection were used in the experiment. The QD labeling process took about $50 \mathrm{~min}$, after which the cells were immediately transferred to a microscope stage for $1 \mathrm{~h}$ imaging. The cell culture, QD labeling, and fluorescent imaging were all performed at room temperature. Because the turnover rate of AChRs in the cell membrane is in days [64], which is much longer than the processing time $(\sim 2 \mathrm{~h})$, the AChRs are biologically stable during the labeling and imaging processes. The QD labeling was conducted at low tag densities to avoid AChR and/or QD clustering and to reduce tracking ambiguities between the consecutive images of the QDs [15].

Typically, we have 200 QDs in a viewing area of $100 \times 100 \mu \mathrm{m}^{2}$ on the bottom side of the membrane facing the substrate. At this low tag density, a typical distance between two adjacent QDs is about $7 \mu \mathrm{m}$, which is more than 300 times larger than the QD radius, and thus QD clustering (and hence endocytosis of AChR clusters) is effectively removed $[15,63]$. Xenopus muscle cells in the primary culture are static with no visible movement during the experiment, and they have a large area for optical observation. This is an optimal condition that we choose for tracking a large volume of AChR trajectories.

\section{Single-particle tracking and construction of potential landscape}

Single-particle tracking (SPT) was performed using a homemade tracking program written in MATLAB, which is 
based on the standard tracking algorithm $[13,15,65,66]$. With this advanced SPT algorithm, we were able to obtain the AChR position $\mathbf{r}(t)$ at time $t$, and the AChR trajectories were constructed from the consecutive images. From the movie of stationary QDs (stuck on a glass coverslip), we found that their displacement over a time period of $200 \mathrm{~s}$ is less than $24 \mathrm{~nm}(=0.48$ pixel $)$. This result sets the accuracy of the measurement of the two-dimensional (2D) displacement vector, $\Delta \mathbf{r}(\tau)=\mathbf{r}(t+\tau)-\mathbf{r}(t)$, where $\tau(\lesssim 300 \mathrm{~s})$ is the largest delay time used in the experiment. During this delay time, AChRs have moved several micrometers.

To study the energetics and dynamics of AChRs, we first selected the mobile trajectories from the whole set of AChR trajectories. This is done by computing the radius of gyration $R_{g}(\tau)$ of each AChR trajectory obtained over a time period of $\tau$,

$$
R_{g}^{2}(\tau)=\frac{1}{N} \sum_{i=1}^{N}\left[\left(x_{i}-\langle x\rangle\right)^{2}+\left(y_{i}-\langle y\rangle\right)^{2}\right],
$$

where $N$ is the total number of time steps in each trajectory, $x_{i}$ and $y_{i}$ are the projection of the position of each trajectory step on the $x$ - and $y$-axis, respectively, and $\langle x\rangle$ and $\langle y\rangle$ are their mean values. Physically, $R_{g}$ quantifies the size of an AChR trajectory generated during the time lapse $\tau$. A cutoff value of $\left(R_{g}^{\prime}\right)_{c}=0.3$ was used in the experiment [13], below which the AChR trajectories are treated as immobile ones. Here $R_{g}^{\prime}=R_{g} /\left\langle R_{g}\right\rangle$ is the normalized radius of gyration, with $\left\langle R_{g}\right\rangle$ being the mean value of $R_{g}$. To avoid the effect of cell boundaries on the motion of AChRs, the mobile trajectories that are located in the boundary region were removed from the statistical analysis.

There are two special issues associated with the AChR tracking. One is the nonergodic behavior between the timeand space-averaged quantities, such as the $\operatorname{MSD}\left\langle\Delta \mathbf{r}^{2}(\tau)\right\rangle$, which has been observed for a number of proteins in live cells $[67,68]$. In a recent study of the motion of AChRs for cells cultured on a flat coverslip [13], it was found that the immobile trajectories play a dominant role in determining the value of $\left\langle\Delta \mathbf{r}^{2}(\tau)\right\rangle$. The measured $\left\langle\Delta \mathbf{r}^{2}(\tau)\right\rangle$ for the immobile AChRs is about two orders of magnitude smaller than the value of $\left\langle\Delta \mathbf{r}^{2}(\tau)\right\rangle$ in the long-time regime $(\tau \gtrsim 4 \mathrm{~s})$ for the mobile AChRs, and thus it contributes many near-zero values to the ensemble average. Consequently, the immobile trajectories may play an essential role in determining the difference between the time- and space-averaged $\left\langle\Delta \mathbf{r}^{2}(\tau)\right\rangle$, because the immobile trajectories are typically included in the space-averaged $\left\langle\Delta \mathbf{r}^{2}(\tau)\right\rangle$, whereas in the time-averaged $\left\langle\Delta \mathbf{r}^{2}(\tau)\right\rangle$, one usually only samples the mobile trajectories [67-69]. For these reasons, we removed the immobile trajectories from the ensemble average as mentioned above, so that the measured $\left\langle\Delta \mathbf{r}^{2}(\tau)\right\rangle$ under different sample conditions remains the same [13].

The other issue is the blinking effect of the QDs [70]. The fluorescent intensity of the QDs fluctuates constantly and becomes invisible intermittently. This blinking effect causes a breakup of some continuous trajectories and thus reduces the accuracy of the long-time tracking of AChRs. The broken trajectories can be relinked using a searching algorithm in Ref. [65]. The algorithm was originally designed to relink the broken trajectories of those colloid particles moving in and out of the observation area. It applies well here to reconnect the fragmental trajectories of QDs [13]. To identify the next position of a QD from a previous frame, a search radius $r$ is carefully chosen using the diffusion radius $r=\left(D_{L} \tau_{0}\right)^{1 / 2}$ as an estimate of the upper limit of a single step displacement, where $D_{L}$ is the long-time diffusion coefficient of the AChRs, and $\tau_{0}$ is the time interval between the two adjacent frames. The program then attempts to find the nearest QD within the defined radius $r$. If no QD is present in the searching area, the QD is missing either permanently or temporarily because of the QD blinking. A record of missing QD counts is set up in the tracking program to retrieve those missing QDs within a time period of $1 \mathrm{~s}$ (within 5 frames at $5 \mathrm{fps}$ ). This relinking procedure increases the tracking length of the AChR trajectories and thus enhances the accuracy of their long-time statistics.

For the cells cultured on the microwell-patterned substrate, the AChR trajectories typically occupy multiple potential traps that are defined by the microwell pattern. To further improve the statistical accuracy of $H(x, y)$, we take advantage of the spatial periodicity of $H(x, y)$ and divide the measured $H(x, y)$ over the entire area into unit cells, each containing only one peak at the cell center. The measured $H(x, y)$ 's in these unit cells are added together after a proper alignment, and we obtain the final $H(x, y)$ over a single potential trap with the highest statistical accuracy.

Similarly, for the cells cultured on the microgroovepatterned substrate, we first overlay the AChR trajectories on top of the bright field image of the microgrooves, and we define the direction along the microgrooves as the $y$-axis, so that the resulting $H(x, y)=H(x)$ depends only on the $x$ position across the microgrooves. The individual trajectories are then divided into different grooves. The measured $H(x)$ 's for different grooves are added together after a proper alignment, and we obtain the final $H(x)$ over a single groove with the highest statistical accuracy. With the large volume of AChR trajectories, as shown in Table I, we obtained the trapping potentials $U(x, y)$ for a microwell and $U(x)$ for a microgroove with an accuracy better than $0.1 k_{B} T$.

For quasi-1D motion of AChRs across the microgroove, their MSD is defined as

$$
\left\langle\Delta x^{2}(\tau)\right\rangle_{y}=\left\langle[x(t+\tau)-x(t)]^{2}\right\rangle_{y}=2 D_{L} \tau,
$$

where $\langle\cdots\rangle_{y}$ denotes an average over all values of $y$, and the long-time diffusion coefficient $D_{L}$ is obtained by fitting the MSD data at large values of delay time $\tau$ (>40 s) to the last equality of Eq. (9). The local diffusion coefficients, $D_{x}(x)$ and $D_{y}(x)$, and local drift velocities, $V_{x}(x)$ and $V_{y}(x)$, at the shortest delay time $\tau=0.2 \mathrm{~s}$ are defined as

$$
D_{x}(x)=\frac{\left\langle\Delta x^{2}(\tau)\right\rangle}{2 \tau}, \quad D_{y}(x)=\frac{\left\langle\Delta y^{2}(\tau)\right\rangle}{2 \tau},
$$

and

$$
V_{x}(x)=\frac{\langle\Delta x(\tau)\rangle}{\tau}, \quad V_{y}(x)=\frac{\langle\Delta y(\tau)\rangle}{\tau} .
$$

More details about the cell treatments and sample sizes used in this study are summarized in Table I. To further build up the statistics, we typically track AChRs from more than 16 cells 
TABLE I. Summary of cell treatments and sample sizes. The columns, from left to right, list the type of substrates used, the treatments to the live muscle cells, the number of cells imaged, and the total number of trajectories used in the statistical analysis, respectively.

\begin{tabular}{llcc}
\hline \hline Substrate pattern & \multicolumn{1}{c}{ Cell treatment } & No. of cells & No. of trajectories \\
\hline Microgroove $(\lambda=2 \mu \mathrm{m})$ & QD labelling & 65 & 25772 \\
Flat substrate & QD labelling & 18 & 4791 \\
Microgroove $(\lambda=2 \mu \mathrm{m})$ & Matrigel treatment & 39 & 8321 \\
Microgroove $(\lambda=2 \mu \mathrm{m})$ & No Matrigel treatment (control) & 18 & 12481 \\
Microgroove $(\lambda=2 \mu \mathrm{m})$ & ATP depletion & 32 & 10068 \\
Microgroove $(\lambda=2 \mu \mathrm{m})$ & No ATP depletion (control) & 30 & 4211 \\
Microgroove $(\lambda=2 \mu \mathrm{m})$ & Blebbistatin treatment & 43 & 5116 \\
Microgroove $(\lambda=2 \mu \mathrm{m})$ & No Blebbistatin treatment (control) & 16 & 3011 \\
Microgroove $(\lambda=3 \mu \mathrm{m})$ & Matrigel treatment & 30 & 2473 \\
Microgroove $(\lambda=3 \mu \mathrm{m})$ & No Matrigel treatment (control & 47 & 5979 \\
Microwell $(\mathrm{d}=2 \mu \mathrm{m})$ & QD labelling & 28 & 7970 \\
Microwell $(\mathrm{d}=3 \mu \mathrm{m})$ & QD labelling & 19 & 5675 \\
& & 385 & 95868 \\
\hline \hline
\end{tabular}

cultured under the same condition, so that more than $4 \times 10^{3}$ trajectories were used for statistical analysis.

\section{ACKNOWLEDGMENTS}

The authors wish to thank C. Lee and A.C.K. Tse for providing Xenopus embryos for the ATP depletion experiments, D. Guan and C. Yan for their help in the AFM measurements, and Y. Ding for his help in early experiments on microgroove patterning. This work was supported in part by RGC of Hong Kong under Grants No. 16306418 (P.T.), No. 16302718 (P.T.), No. 16300421 (P.T.), and No. 16102417 (P.H.) and by MoST of Taiwan under Grants No. 110-2112-M-008-026MY3 (P.Y.L.) and No. 110-2112-M-008-030- (H.Y.C.). P.Y.L. and H.Y.C. also acknowledge support by NCTS of Taiwan.

\section{APPENDIX: THEORETICAL MODEL}

\section{Potential field generated by anchored proteins}

To quantitatively describe the potential field $U(x)$ generated by anchored integrins, we consider a medium containing fixed obstacles (anchored integrins) which (i) prevent a protein molecule (AChR) from passing through (due to a hardcore repulsion) and (ii) form a temporary bound state with the protein molecule (due to mutual attraction). Let $H\left(x, t ; x_{0}, t_{0}\right)$ ( $\equiv H(x, t)$ for short) be the probability density function (PDF) of finding an AChR at the position $x$ and time $t$, whose initial space-time position is $x_{0}$ and $t_{0}$. The PDF $H(x, t)=$ $p_{f}(x, t)+p_{b}(x, t)$ contains two sub-populations with $p_{f}(x, t)$ being the PDF that a protein is located at $x$ and is free to diffuse at $t$ and $p_{b}(x, t)$ being the PDF that a protein is located at $x$ and is in a bound state with an obstacle at $t$.
The binding and unbinding of a protein with an obstacle can be described by a simple two-state dynamics,

$$
\frac{\partial p_{b}}{\partial t}=k_{b} n p_{f}-k_{u} p_{b}
$$

where $n(x)$ is the density (area fraction) of the obstacles, and $k_{b}$ and $k_{u}$ are, respectively, the binding and unbinding rates. On the other hand, the evolution of $p_{f}$ has to include the contribution from protein diffusion,

$$
\frac{\partial p_{f}}{\partial t}=-k_{b} n p_{f}+k_{u} p_{b}+S,
$$

where $S(x, t)$ satisfies

$$
\begin{aligned}
S(x, t) d t= & -\left(\frac{1-c(x+d x)}{2}+\frac{1-c(x-d x)}{2}\right) p_{f}(x, t) \\
& +\frac{1-c(x)}{2} p_{f}(x+d x, t) \\
& +\frac{1-c(x)}{2} p_{f}(x-d x, t) .
\end{aligned}
$$

Here $c(x)$ represents how obstacles at $x$ hinder the motion of free proteins. Intuitively, $c(x)$ is proportional to $n(x)$, i.e., $c(x) \simeq n(x)$. By taking $d x \rightarrow 0$, we find

$$
S(x, t)=D_{0} \frac{\partial}{\partial x}\left[[1-n(x)] \frac{\partial p_{f}}{\partial x}+\frac{d n(x)}{d x} p_{f}\right],
$$

where $D_{0}=d x^{2} / 2 d t$.

In the long-time limit $t \gg 1 / k_{b}$ and $t \gg 1 / k_{u}$, we have

$$
k_{b} n p_{f}=k_{u} p_{b} \text {. }
$$

This leads to

$$
p_{f}=\frac{H}{1+k_{b} n / k_{u}} .
$$


The equation for $H(x, t)$ can be obtained by first summing over Eqs. (A1) and (A2) and then substituting Eqs. (A3) and (A5),

$$
\begin{gathered}
\frac{\partial H(x, t)}{\partial t}=D_{0} \frac{\partial}{\partial x}\left[\left(\frac{d n(x) / d x}{1+\alpha n(x)}-\frac{\alpha[1-n(x)] d n(x) / d x}{[1+\alpha n(x)]^{2}}\right)\right. \\
\left.H(x, t)+\frac{1-n(x)}{1+\alpha n(x)} \frac{\partial H(x, t)}{\partial x}\right],
\end{gathered}
$$

where $\partial H(x, t) / \partial t=S$ and $\alpha=k_{b} / k_{u}$.

At equilibrium, we have

$$
\begin{gathered}
\left(\frac{d n(x) / d x}{1+\alpha n(x)}-\frac{\alpha[1-n(x)] d n(x) / d x}{[1+\alpha n(x)]^{2}}\right) \\
\quad \times H(x)+\frac{1-n(x)}{1+\alpha n(x)} \frac{d H(x)}{d x}=0 .
\end{gathered}
$$

It is straightforward to obtain the equilibrium distribution

$$
H(x)=H_{0}[1-n(x)][1+\alpha n(x)],
$$

where $H_{0}$ is an integration constant. The equilibrium PDF $H(x)$ is related to the potential $U(x)$ by the Boltzmann distribution [34,35],

$$
\begin{aligned}
U(x) & \equiv-k_{B} T \ln H(x) \\
& =U_{0}-k_{B} T \ln ([1-n(x)][1+\alpha n(x)]),
\end{aligned}
$$

where $U_{0}=-k_{B} T \ln H_{0}$. Equation (A9) is given in the main text as Eq. (3). Since the decrease of entropy of a mobile protein in the presence of fixed obstacles is simply $k_{B} \ln [1-$ $n(x)$ ], Eq. (A9) can be understood as the increase in free energy of the mobile proteins due to the entropic force from the anchored integrins. For small values of $n(x)$ and $\alpha=0$, Eq. (A9) becomes

$$
U(x) \simeq n(x) k_{B} T,
$$

where $U_{0}$ is chosen in such a way that $U(x)=0$ when $n(x)=$ 0 . The physical meaning of Eq. (A10) is explained in the main text.

\section{Protein dynamics in the potential field $U(x)$}

While it is convenient to derive the potential field $U(x)$ from the lattice model as discussed above, a more general model for the actual dynamics of AChRs in the potential field generated by anchored integrins may be needed, as the simple lattice model does not take other complex processes in the cells into account. In general, the total flux of AChRs can be expressed as

$$
J(x, t)=-\left[\frac{d U(x)}{d x} \frac{H(x, t)}{\xi(x)}+D(x) \frac{\partial H(x, t)}{\partial x}\right],
$$

where $\xi(x)$ and $D(x)$ are, respectively, the friction and diffusion coefficients of mobile AChRs in the $x$-direction; both are functions of $x$. The Smoluchowski equation for $H(x, t)$ reads [44]

$$
\begin{aligned}
\frac{\partial H(x, t)}{\partial t} & =-\frac{\partial J(x, t)}{\partial x} \\
& =\frac{\partial}{\partial x}\left[\frac{d U(x)}{d x} \frac{H(x, t)}{\xi(x)}+D(x) \frac{\partial H(x, t)}{\partial x}\right]
\end{aligned}
$$

The zero-flux steady-state distribution of $H(x)$ is obtained by solving $J=0$, and the result is

$$
\ln H(x)=-\int_{0}^{x} d u \frac{U^{\prime}(u)}{\xi(u) D(u)}+C,
$$

where $U^{\prime}(u)=d U(u) / d u$, and $C$ is an integration constant. With the effective potential defined as $U_{\text {eff }}(x) \equiv$ $-k_{B} T \ln H(x)$, we find from (A13) that the derivative of $U_{\text {eff }}$ is given by

$$
U_{\mathrm{eff}}^{\prime}(x)=k_{B} T \frac{U^{\prime}(x)}{\xi(x) D(x)} .
$$

Equation (A14) states that in general the effective potential $U_{\text {eff }}(x)$ is not the same as the actual potential $U(x)$ unless the local Stokes-Einstein relation, $\xi(x) D(x)=k_{B} T$, is satisfied. The proper reconstruction formula for the actual potential is

$$
U(x)=U(0)-\int_{0}^{x} d u \frac{\xi(u) D(u) H^{\prime}(u)}{H(u)},
$$

where $H^{\prime}(u)=d H(u) / d u$. If the Stokes-Einstein relation holds locally, Eq. (A14) reduces to $U_{\text {eff }}^{\prime}(x)=U^{\prime}(x)$, i.e., there is no distinction between the effective and actual potentials apart from an arbitrary additive constant. In this case, the equilibrium distribution $H(x)$ is of a Boltzmann distribution.

Next, we calculate the first and second moments, $\langle\Delta x\rangle$ and $\left\langle(\Delta x)^{2}\right\rangle$, of AChR's displacements that can be measured directly in the experiment. The AChR is initially at $x_{0}$ and its location at a (short) time $\tau$ later is $x$, so that the displacement $\Delta x(\tau)=x-x_{0}$. For an initial distribution $\delta\left(x-x_{0}\right)$ and a small $\tau$, Eq. (A12) gives

$$
\begin{aligned}
H(x, \tau) & \simeq\left(1+\tau \partial_{t}\right) \delta\left(x-x_{0}\right) \\
& =\left[1+\tau \partial_{x}\left(\frac{U^{\prime}(x)}{\xi(x)}+D(x) \partial_{x}\right)\right] \delta\left(x-x_{0}\right) .
\end{aligned}
$$

Then integrating by parts twice, one gets

$$
\begin{aligned}
\frac{\langle\Delta x\rangle}{\tau} & =\frac{1}{\tau} \int d x H(x, \tau)\left(x-x_{0}\right) \\
& \simeq \int d x\left(x-x_{0}\right) \partial_{x}\left[\frac{U^{\prime}(x)}{\xi(x)}+D(x) \partial_{x}\right] \delta\left(x-x_{0}\right) \\
& =-\frac{U^{\prime}\left(x_{0}\right)}{\xi\left(x_{0}\right)}-\int d x D(x) \partial_{x} \delta\left(x-x_{0}\right) \\
& =-\frac{U^{\prime}\left(x_{0}\right)}{\xi\left(x_{0}\right)}+D^{\prime}\left(x_{0}\right)
\end{aligned}
$$

Similarly,

$$
\begin{aligned}
\frac{\left\langle\Delta x^{2}\right\rangle}{\tau} & =\frac{1}{\tau} \int d x H(x, \tau)\left(x-x_{0}\right)^{2} \\
& \simeq \int d x\left(x-x_{0}\right)^{2} \partial_{x}\left[\frac{U^{\prime}(x)}{\xi(x)}+D(x) \partial_{x}\right] \delta\left(x-x_{0}\right) \\
& =2 D\left(x_{0}\right) .
\end{aligned}
$$

We thus obtain the local drift velocity $V\left(x_{0}\right)$ and diffusion coefficient $D\left(x_{0}\right)$ from the experimentally measured 
short-time $\langle\Delta x\rangle$ and $\left\langle(\Delta x)^{2}\right\rangle$ as a function of the initial location $x_{0}$,

$$
\begin{gathered}
\frac{\langle\Delta x\rangle}{\tau} \equiv V\left(x_{0}\right) \simeq-U^{\prime}\left(x_{0}\right) / \xi\left(x_{0}\right)+D^{\prime}\left(x_{0}\right), \\
\frac{\left\langle\Delta x^{2}\right\rangle}{2 \tau} \simeq D\left(x_{0}\right) .
\end{gathered}
$$

Equation (A19) gives the correction of the terminal velocity, $-U^{\prime}\left(x_{0}\right) / \xi\left(x_{0}\right)$, due to the spatially nonuniform diffusion coefficient. Notice that Eqs. (A19) and (A20) hold regardless of whether the Stokes-Einstein relation holds or not. For the case of passive diffusion in which the Stokes-Einstein relation holds, Eq. (A19) can be rewritten as

$$
V(x)=-D(x) U^{\prime}(x) /\left(k_{B} T\right)+D^{\prime}(x),
$$

which is the first equality of Eq. (5) in the main text.

Finally, substituting Eq. (A19) into Eq. (A14) and integrating, we have

$$
U_{\text {eff }}(x)=U_{\text {eff }}(0)-k_{B} T \int_{0}^{x} \frac{V(u)-d D(u) / d u}{D(u)} d u .
$$

Equation (A22) is Eq. (6) in the main text, which we applied to obtain the effective trapping potential shown in Fig. 3(d).

It is also interesting to check, besides providing the potential field, if the simple lattice model in Appendix 1 happens to provide a quantitative description of $\mathrm{AChR}$ dynamics. By comparing Eq. (A6) with Eq. (A12), we find the diffusion constant $D_{l}(x)$ of the lattice model

$$
D_{l}(x)=D_{0} \frac{1-n(x)}{1+\alpha n(x)},
$$

with

$$
D_{0}(1-\alpha) n^{\prime}(x)=\frac{U^{\prime}(x)}{\xi(x)} .
$$

Equation (A23) is given in the main text as Eq. (4).

Substituting Eqs. (A23) and (A24) into Eq. (A19), we obtain the local drift velocity $V_{l}(x)$ based on the lattice model,

$$
V_{l}(x)=-2 D_{0} \frac{n^{\prime}(x)}{1+\alpha n(x)},
$$

which is the second equality of Eq. (5) in the main text. As shown in Figs. 3(e) and 3(f), although Eq. (4) and the second equality of Eq. (5) agree with the measured $D(x)$ and $V_{x}(x)$ qualitatively, they do not agree with experimental data quantitatively. This is because the dynamics of AChRs in the potential field are also influenced by small concentration gradients of other (unknown) anchored proteins, which are not included in the lattice model.
[1] F. Höfling and T. Franosch, Anomalous transport in the crowded world of biological cells, Rep. Prog. Phys. 76, 046602 (2013).

[2] A. Triller and D. Choquet, New concepts in synaptic biology derived from single-molecule imaging, Neuron 59, 359 (2008).

[3] D. A. Rusakov, L. P. Savtchenko, K. Zheng, and J. M. Henley, Shaping the synaptic signal: molecular mobility inside and outside the cleft, Trends Neurosci. 34, 359 (2011).

[4] C. P. Brangwynne, G. H. Koenderink, F. C. MacKintosh, and D. A. Weitz, Intracellular transport by active diffusion, Trends Cell Biol. 19, 423 (2009).

[5] K. Jacobson, P. Liu, and B. C. Lagerholm, The lateral organization and mobility of plasma membrane components, Cell 177, 806 (2019).

[6] S. Stam and M. L. Gardel, Cutting through the noise: the mechanics of intracellular transport, Dev. Cell 30, 365 (2014).

[7] J. L. Ross, M. Y. Ali, and D. M. Warshaw, Cargo transport: molecular motors navigate a complex cytoskeleton, Curr. Opin. Cell Biol. 20, 41 (2008).

[8] D. Wang, L. She, Y. N. Sui, X. B. Yuan, Y. Wen, and M. M. Poo, Forward transport of proteins in the plasma membrane of migrating cerebellar granule cells, Proc. Natl. Acad. Sci. (USA) 109, E3558 (2012).

[9] Y. H. Tee et al., Cellular chirality arising from the selforganization of the actin cytoskeleton, Nat. Cell Biol. 17, 445 (2015).

[10] A. Kusumi et al., Dynamic organizing principles of the plasma membrane that regulate signal transduction: commemorating the fortieth anniversary of Singer and Nicolson's fluid-mosaic model, Annu. Rev. Cell Dev. Biol. 28, 215 (2012).
[11] W. S. Trimble and S. Grinstein, Barriers to the free diffusion of proteins and lipids in the plasma membrane, J. Cell Biol. 208 259 (2015)

[12] C. Leterrier, The axon initial segment: an updated viewpoint, J. Neurosci. 38, 2135 (2018).

[13] W. He et al., Dynamic heterogeneity and non-Gaussian statistics for acetylcholine receptors on live cell membrane, Nat. Commun. 7, 11701 (2016).

[14] Z. Sun, M. Costell, and R. Fässler, Integrin activation by talin, kindlin and mechanical forces, Nat. Cell Biol. 21, 25 (2019).

[15] W. He, Y. Su, H. B. Peng, and P. Tong, Dynamic heterogeneity and non-Gaussian statistics for ganglioside GM1s and acetylcholine receptors on live cell membrane, Mol. Biol. Cell 31, 1380 (2020).

[16] Z. Shi, Z. T. Graber, T. Baumgart, H. A. Stone, and A. E. Cohen, Cell membranes resist flow, Cell 175, 1769 (2018).

[17] M. J. Saxton, Lateral diffusion in an archipelago. The effect of mobile obstacles, Biophys. J. 52, 989 (1987).

[18] M. A. Deverall et al., Membrane lateral mobility obstructed by polymer-tethered lipids studied at the single molecule level, Biophys. J. 88, 1875 (2005).

[19] J. Tsai, E. Sun, Y. Gao, J. C. Hone, and L. C. Kam, NonBrownian diffusion of membrane molecules in nanopatterned supported lipid bilayers, Nano Lett. 8, 425 (2008).

[20] M. Renner, D. Choquet, and A. Triller, Control of the postsynaptic membrane viscosity, J. Neurosci. 29, 2926 (2009).

[21] E. Sevcsik, M. Brameshuber, M. Fölser, J. Weghuber, A. Honigmann, and G. J. Schütz, GPI-anchored proteins do not reside in ordered domains in the live cell plasma membrane, Nat. Commun. 6, 6969 (2015). 
[22] R. Diez-Ahedo, D. Normanno, O. Esteban, G. J. Bakker, C. G. Figdor, A. Cambi, and M. F. Garcia-Parajo, Dynamic re-organization of individual adhesion nanoclusters in living cells by ligand-patterned surfaces, Small 5, 1258 (2009).

[23] T. Auth and N. S. Gov, Diffusion in a fluid membrane with a flexible cortical cytoskeleton, Biophys. J. 96, 818 (2009).

[24] T. K. Fujiwara et al., Confined diffusion of transmembrane proteins and lipids induced by the same actin meshwork lining the plasma membrane, Mol. Biol. Cell 27, 1101 (2016).

[25] A. L. York and J. Q. Zheng, Super-resolution microscopy reveals a nanoscale organization of acetylcholine receptors for trans-synaptic alignment at neuromuscular synapses, eNeuro $\mathbf{4}$, 0232 (2017).

[26] C. Nakada et al., Accumulation of anchored proteins forms membrane diffusion barriers during neuronal polarization, Nat. Cell Biol. 5, 626 (2003).

[27] S. A. Freeman et al., Integrins form an expanding diffusional barrier that coordinates phagocytosis, Cell 164, 128 (2016).

[28] J. R. Sanes and J. W. Lichtman, Development of the vertebrate neuromuscular junction, Annu. Rev. Neurosci. 22, 389 (1999).

[29] M. Théry, Micropatterning as a tool to decipher cell morphogenesis and functions, J. Cell Sci. 123, 4201 (2010).

[30] L. Geng, H. L. Zhang, and H. B. Peng, The formation of acetylcholine receptor clusters visualized with quantum dots, BMC Neurosci. 10, 80 (2009).

[31] H. L. Zhang and H. B. Peng, Mechanism of acetylcholine receptor cluster formation induced by DC electric field, PLoS One 6, e26805 (2011).

[32] D. Alcor, G. Gouzer, and A. Triller, Single-particle tracking methods for the study of membrane receptors dynamics, Eur. J. Neurosci. 30, 987 (2009).

[33] O. Rossier et al., Integrins $\beta 1$ and $\beta 3$ exhibit distinct dynamic nanoscale organizations inside focal adhesions, Nat. Cell Biol. 14, 1057 (2012).

[34] S. Jin, P. M. Haggie, and A. S. Verkman, Single-particle tracking of membrane protein diffusion in a potential: simulation, detection, and application to confined diffusion of $\mathrm{CFTR} \mathrm{Cl}^{-}$ channels, Biophys. J. 93, 1079 (2007).

[35] X. G. Ma, P. Y. Lai, and P. Tong, Colloidal diffusion over a periodic energy landscape, Soft Matter 9, 8826 (2013).

[36] Y. Su et al., Colloidal diffusion over a quasicrystalline-patterned surface, J. Chem. Phys. 146, 214903 (2017).

[37] R. D. Hanes, C. Dalle-Ferrier, M. Schmiedeberg, M. C. Jenkins, and S. U. Egelhaaf, Colloids in one dimensional random energy landscapes, Soft Matter 8, 2714 (2010).

[38] A. E. Rodríguez-Fraticelli, M. Auzan, M. A. Alonso, M. Bornens, and F. Martín-Belmonte, Cell confinement controls centrosome positioning and lumen initiation during epithelial morphogenesis, J. Cell Biol. 198, 1011 (2012).

[39] Y. Shen et al., Mechanical characterization of microengineered epithelial cysts by using atomic force microscopy, Biophys. J. 112, 398 (2017).

[40] N. Akhtar and C. H. Streuli, An integrin-ILK-microtubule network orients cell polarity and lumen formation in glandular epithelium, Nat. Cell Biol. 15, 17 (2013).

[41] See Supplemental Material at http://link.aps.org/supplemental/ 10.1103/PhysRevResearch.3.043195 for supplemental figures, which complement the main text.
[42] F. Kong, A. J. García, A. P. Mould, M. J. Humphries, and C. Zhu, Demonstration of catch bonds between an integrin and its ligand, J. Cell Biol. 185, 1275 (2009).

[43] M. Guo et al., Probing the stochastic, motor-driven properties of the cytoplasm using force spectrum microscopy, Cell 158, 822 (2014).

[44] M. Doi, Soft Matter Physics (Oxford University Press, Oxford, 2013).

[45] D. J. Burkin, J. E. Kim, M. Gu, and S. J. Kaufman, Laminin and $\alpha 7 \beta 1$ integrin regulate agrin-induced clustering of acetylcholine receptors, J. Cell Sci. 113, 2877 (2000).

[46] C. Charrier et al., A crosstalk between $\beta 1$ and $\beta 3$ integrins controls glycine receptor and gephyrin trafficking at synapses, Nat. Neurosci. 13, 1388 (2010).

[47] J. B. Masson et al., Mapping the energy and diffusion landscapes of membrane proteins at the cell surface using high-density single-molecule imaging and Bayesian inference: application to the multiscale dynamics of glycine receptors in the neuronal membrane, Biophys. J. 106, 74 (2014).

[48] T. Sungkaworn et al., Single-molecule imaging reveals receptor-G protein interactions at cell surface hot spots, Nature (London) 550, 543 (2017).

[49] T. Boiko, M. Vakulenko, H. Ewers, C. C. Yap, C. Norden, and B. Winckler, Ankyrin-dependent and -independent mechanisms orchestrate axonal compartmentalization of L1 family members neurofascin and L1/neuron-glia cell adhesion molecule, J. Neurosci. 27, 590 (2007).

[50] S. L. Jones and T. M. Svitkina, Axon initial segment cytoskeleton: architecture, development, and role in neuron polarity, Neural Plast. 2016, 6808293 (2016).

[51] H. Wu, W. C. Xiong, and L. Mei, To build a synapse: signaling pathways in neuromuscular junction assembly, Development 137, 1017 (2010).

[52] C. Edwards and H. L. Frisch, A model for the localization of acetylcholine receptors at the muscle endplate, J. Neurobiol. 7, 377 (1976).

[53] M. M. Poo, Rapid lateral diffusion of functional ACh receptors in embryonic muscle cell membrane, Nature (London) 295, 332 (1982).

[54] M. Sheng and E. Kim, The postsynaptic organization of synapses, Cold Spring Harbor Perspect. Biol. 3, a005678 (2011).

[55] M. Zeng et al., Reconstituted postsynaptic density as a molecular platform for understanding synapse formation and plasticity, Cell 174, 1172 (2018).

[56] A. A. Hyman, C. A. Weber, and F. Jülicher, Liquid-liquid phase separation in biology, Annu. Rev. Cell Dev. Biol. 30, 39 (2014).

[57] M. Shamir, Y. Bar-On, R. Phillips, and R. Milo, SnapShot: Timescales in cell biology, Cell 164, 1302 (2016).

[58] Y. Ding et al., Directing vascular cell selectivity and hemocompatibility on patterned platforms featuring variable topographic geometry and size, ACS Appl. Mater. Interfaces 6, 12062 (2014).

[59] N. Unwin, Neurotransmitter action: opening of ligand-gated ion channels, Cell 72, 31 (1993).

[60] H. B. Peng, L. P. Baker, and Q. Chen, Tissue culture of Xenopus neurons and muscle cells as a model for studying synaptic induction, Methods Cell Biol. 36, 511 (1991). 
[61] M. W. Cohen, B. G. Hoffstrom, and D. W. DeSimone, Active zones on motor nerve terminals contain $\alpha 3 \beta 1$ integrin, J. Neurosci. 20, 4912 (2000).

[62] H. Bannai, S. Lévi, C. Schweizer, M. Dahan, and A. Triller, Imaging the lateral diffusion of membrane molecules with quantum dots, Nat. Protoc. 1, 2628 (2006).

[63] L. Geng, Visualization of nicotinic acetylcholine receptor trafficking with quantum dots in Xenopus muscle cells. Ph.D. thesis, HKUST (2006).

[64] Z. Z. Wang, A. Mathias, M. Gautam, and Z. W. Hall, Metabolic stabilization of muscle nicotinic acetylcholine receptor by rapsyn, J. Neurosci. 19, 1998 (1999).

[65] J. C. Crocker and D. G. Grier, Methods of digital video microscopy for colloidal studies, J. Colloid Interface Sci. 179, 298 (1996).
[66] S. Anthony, L. Zhang, and S. Granick, Methods to track singlemolecule trajectories, Langmuir 22, 5266 (2006).

[67] A. V. Weigel, B. Simon, M. M. Tamkun, and D. Krapf, Ergodic and nonergodic processes coexist in the plasma membrane as observed by single-molecule tracking, Proc. Natl. Acad. Sci. (USA) 108, 6438 (2011).

[68] J.-H. Jeon, V. Tejedor, S. Burov, E. Barkai, C. UnkelSelhuber, K. Berg-Sørensen, L. Oddershede, and R. Metzier, In Vivo Anomalous Diffusion and Weak Ergodicity Breaking of Lipid Granules, Phys. Rev. Lett. 106, 048103 (2011).

[69] I. Golding and E. C. Cox, Physical Nature of Bacterial Cytoplasm, Phys. Rev. Lett. 96, 098102 (2006).

[70] X. Michalet et al., Quantum dots for live cells, in vivo imaging and diagnostics, Science 307, 538 (2005). 